\title{
Gravity-mode period spacings and near-core rotation rates of $611 \gamma$ Doradus stars with Kepler
}

\author{
Gang $\mathrm{Li}^{1,3^{\star}}$, Timothy Van Reeth ${ }^{1,2,3}$, Timothy R. Bedding ${ }^{1,3 \dagger}$, Simon J. Murphy ${ }^{1,3}$, \\ ${\text { Victoria } \text { Antoci }^{3} \text {, Rhita-Maria Ouazzani }}^{4}$, Nicholas H. Barbara ${ }^{1,3}$ \\ ${ }^{1}$ Sydney Institute for Astronomy (SIfA), School of Physics, 2006 University of Sydney, Australia \\ ${ }^{2}$ Institute of Astronomy, KU Leuven, Celestijnenlaan 200D, B-3001 Leuven, Belgium \\ ${ }^{3}$ Stellar Astrophysics Centre, Department of Physics and Astronomy, Aarhus University, Ny Munkegade 120, DK-8000 Aarhus C, Denmark \\ ${ }^{4}$ LESIA, Observatoire de Paris, PSL Research University, CNRS, Sorbonne Universités, UPMC Univ. Paris 06, Univ. Paris Diderot, \\ Sorbonne Paris Cité, 5 place Jules Janssen, 92195 Meudon, France
}

Accepted XXX. Received YYY; in original form ZZZ

\begin{abstract}
We report our survey of $\gamma$ Dor stars from the 4-yr Kepler mission. These stars pulsate mainly in g modes and $\mathrm{r}$ modes, showing period-spacing patterns in the amplitude spectra. The period-spacing patterns are sensitive to the chemical composition gradients and the near-core rotation, hence they are essential for understanding the stellar interior. We identified period-spacing patterns in $611 \gamma$ Dor stars. Almost every star pulsates in dipole g modes, while about $30 \%$ of stars also show clear patterns for quadrupole g modes and $16 \%$ of stars present $\mathrm{r}$ mode patterns. We measure periods, period spacings, and the gradient of the period spacings. These three observables guide the mode identifications and can be used to estimate the near-core rotation rate. We find many stars are hotter and show longer period-spacing patterns than theory. Using the Traditional Approximation of Rotation (TAR), we inferred the asymptotic spacings, the near-core rotation rates, and the radial orders of the $\mathrm{g}$ and $\mathrm{r}$ modes. Most stars have a near-core rotation rate around $1 \mathrm{~d}^{-1}$ and an asymptotic spacing around $4000 \mathrm{~s}$. We also find that many stars rotate more slowly than predicted by theory for unclear reasons. 11 stars show rotational splittings with fast rotation rates. We compared the observed slope-rotation relation with the theory and find a large spread. We detected rotational modulations in 58 stars and used them to derive the core-tosurface rotation ratios. The interiors rotate faster than the cores in most stars, but by no more than $5 \%$.
\end{abstract}

Key words: stars: oscillations - stars: rotation - stars: variables

\section{INTRODUCTION}

Rotation affects the transport of chemical elements and angular momentum in stars, so it changes stellar structure and evolution (e.g. Maeder 2009; Mathis et al. 2013). However, the theoretical description of rotation is still a matter of debate. For example, the observed core-to-surface rotation rate ratios in red giants are smaller than predicted by theory (see e.g. Mosser et al. 2012; Eggenberger et al. 2012; Ceillier et al. 2013; Fuller et al. 2019). For A- and F-type main-sequence stars, the typical value of the projected surface rotation velocity is around $100 \mathrm{~km} \mathrm{~s}^{-1}$ and increases with effective temperature (e.g. Fukuda 1982; Groot et al. 1996;

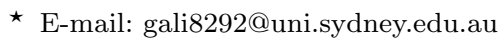

$\dagger$ E-mail: tim.bedding@sydney.edu.au
Abt \& Morrell 1995; Royer et al. 2007). Hence, the effect of rapid rotation must be treated properly.

Stellar oscillations are a powerful tool to investigate the stellar interior. We focus on $\gamma$ Doradus stars, which are Ato F-type main-sequence stars with typical masses from 1.4 to $2.0 \mathrm{M}_{\odot}$ (e.g. Kaye et al. 1999; Van Reeth et al. 2016). The pulsations of $\gamma$ Dor stars are gravity modes with high radial order $(20 \lesssim n \lesssim 100)$ low degree $(l \lesssim 4)$ with typical pulsation period from 0.3 to $3 \mathrm{~d}$ (Balona et al. 1994; Kaye et al. 1999; Saio et al. 2018b; Van Reeth et al. 2018; Li et al. 2019b). Gravity modes have their highest mode energy in the near-core regions (e.g. Triana et al. 2015; Van Reeth et al. 2016). Therefore, $\gamma$ Dor stars allow us to investigate the stellar interior. The excitation mechanism of $\gamma$ Dor stars is still in debate. Guzik et al. (2000); Dupret et al. (2005) reported that the g-mode pulsations are excited by the con- 
vective flux blocking mechanism that operates at the base of the envelope convection zone. Xiong et al. (2016) found that the radiative $\kappa$ mechanism plays a major role in warm $\gamma$ Dor stars while the coupling between convection and oscillations is dominant in cool stars. Turbulent thermal convection is a damping mechanism that gives rise to the red edge of the instability strip. Grassitelli et al. (2015) pointed out that turbulent pressure fluctuations may contribute to the $\gamma$ Dor phenomenon. The instability strip of $\gamma$ Dor stars is located between the solar-like stars and the $\delta$ Scuti stars, overlapping with the red edge of the $\delta$ Scuti instability strip (Dupret et al. 2005; Bouabid et al. 2009, 2013). Hence some $\gamma$ Dor stars show both g- and p-mode oscillations and are called $\delta$ Sct $-\gamma$ Dor hybrids. Pressure modes probe the outer stellar layers, therefore the overall structure along the radial direction can be deduced (e.g. Kurtz et al. 2014; Saio et al. 2015).

Due to the daily aliasing and small amplitudes, the pulsations of $\gamma$ Dor stars were hard to detect with ground-based observations, hence their near-core rotations were unclear for a long time. Thanks to the Kepler space telescope (Koch et al. 2010; Borucki et al. 2010), 4-yr light curves of many stars have been collected. Kurtz et al. (2014) measured the rotational splittings of the $\gamma$ Dor star KIC 11145123, which was the first robust determination of the rotation of the deep core and surface of a main-sequence star. The rotational splittings of $\mathrm{g}$ modes in Slowly Pulsating B (SPB) stars were also reported (e.g. Pápics et al. 2015). For fast rotators, as in the majority of $\gamma$ Dor stars, the period spacings change quasi-linearly with period and can be used to fit the near-core rotation rate (e.g. Van Reeth et al. 2016; Christophe et al. 2018). Now, splittings or period spacings of g modes from tens of $\gamma$ Dor stars were found, both in single stars and binaries, whose rotation profiles are almost uniform (e.g. Triana et al. 2015; Saio et al. 2015; Keen et al. 2015; Van Reeth et al. 2015; Li et al. 2019a; Guo et al. 2017; Kallinger et al. 2017; Li et al. 2019b).

In this paper, we report 960 period-spacing patterns from 611 Kepler $\gamma$ Dor stars, which form the largest sample of identified period-spacing patterns. The period spacing $\Delta P$ is defined as the period difference between two consecutive overtones $\Delta P_{n} \equiv P_{n+1}-P_{n}$ and is a constant in the nonrotating homogeneous stars (according to the asymptotic relation, Shibahashi 1979). The rapid rotation changes stellar structure and frequency values, and the Traditional Approximation of Rotation (TAR), or the complete calculation including the full effect of rotation is necessary to describe the oscillation frequencies more accurately (e.g. Eckart 1960; Lee \& Saio 1987; Townsend 2005; Saio et al. 2018b). Under the TAR, the period spacing decreases with period quasilinearly for the prograde and zonal g modes. Overall, the retrograde $g$ modes have increasing period spacing (Bouabid et al. 2013; Ouazzani et al. 2017) and they are seen in slow rotators but are hard to see in fast rotators ( $\mathrm{Li}$ et al. 2019a; Saio et al. 2018b).

In addition to $\mathrm{g}$ modes and sometimes $\mathrm{p}$ modes, $\gamma$ Dor stars also show Rossby modes (r modes), whose restoring force is the Coriolis force (Papaloizou \& Pringle 1978). Rossby modes propagate retrograde to the rotation direction and have discrete frequencies smaller than the rotation frequency in the corotating reference frame (Saio 1982; Lee \& Saio 1997; Provost et al. 1981). Rossby modes can also be described by the TAR and they also show a quasilinear period-spacing pattern, in which the period spacing increases with period. Using the period-spacing patterns from $\mathrm{g}$ and $\mathrm{r}$ modes, the near-core rotation of tens of $\gamma$ Dor stars were measured to be around $1 \mathrm{~d}^{-1}$ (e.g. Saio et al. 2018a; Van Reeth et al. 2018; Li et al. 2019b). Many of the stars in our sample also show $r$ modes.

We describe our data reduction and TAR fitting in Section 2. Section 3 gives the observation results, including the observed relative occurence rates of different types of modes, the typical structure of the periodogram, the slope-mean period relation of $\gamma$ Dor stars. Section 4 shows the TAR fit results, including the distributions of the asymptotic spacings and the near-core rotation rates, as well as the comparison with the theoretical predictions. Section 5 reveals that the slope-period relation can be used on estimating the nearcore rotation rate. Section 6 reports 11 fast-rotating stars with rotational splittings and Section 7 displays 58 stars with surface rotation modulations. Finally, we conclude our works in Section 8.

\section{DATA ANALYSIS}

We examined 2085 stars with effective temperature between $6000 \mathrm{~K}$ to $10000 \mathrm{~K}$, where we used the input temperatures from the Kepler DR25 data release (Mathur et al. 2017). We found 960 clear period-spacing patterns in 611 stars, including 50 stars by Van Reeth et al. (2015), 22 stars with splittings by Li et al. (2019a), 82 stars with r-mode patterns by Li et al. (2019b), 30 stars by Chowdhury et al. (2018), 44 stars by Murphy et al. (2018), 344 stars found by Barbara et al. (in prep), and the rest we found by visually inspecting light curves and their Fourier transforms (the samples overlap). Barbara et al. (in prep) applied a Gaussian mixture model in a reduced 5D space to classify 12066 stars in the Kepler field. The method involves using a greedy algorithm to select defining features from the HCTSA feature library (Fulcher et al. 2013; Fulcher \& Jones 2017).

We used 4-year Kepler long-cadence light curves (29.45min sampling) from the multi-scale MAP data pipeline (Stumpe et al. 2014). The 4-yr long-cadence data are suitable for $\gamma$ Dor stars since the typical pulsation periods of these stars are around 1 day with period spacings around $1000 \mathrm{~s}$, which require a long observation span to resolve the modes. However, 97 of our stars also have short-cadence data. These data can be used to readily investigate the pressure modes if they are $\gamma$ Dor $-\delta$ Sct hybrids, though thanks to the super-Nyquist asteroseismology technique (Murphy et al. 2013) the Kepler LC data are also sufficient for this purpose.

In each quarter, the light curve was divided by a secondorder polynomial fit to remove any slow trend. We computed the Fourier transform and extracted the frequencies until the signal to noise ratio $(\mathrm{S} / \mathrm{N})$ was smaller than 3 .

The period-spacing patterns were identified by the cross-correlation algorithm described by Li et al. (2019a) and inspected visually. We present the period-spacing patterns of KIC 7694191 as an example in Fig. 1. Figure 1a shows the periodogram, where the locations of peaks for each pattern are shown with dashed vertical lines. We found two periodspacing patterns around $0.20 \mathrm{~d}$ and $0.38 \mathrm{~d}$. The right pattern 

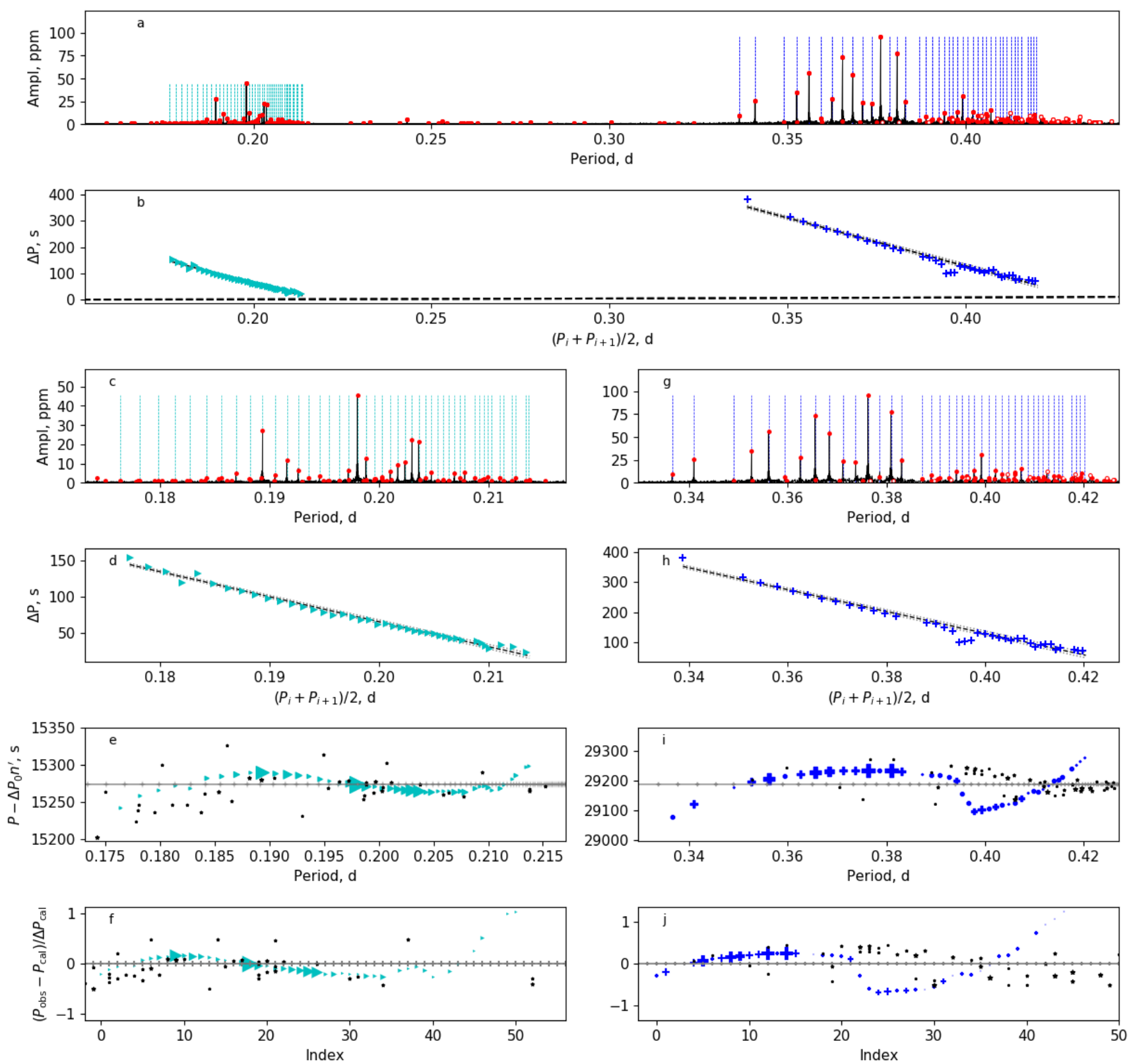

Figure 1. The g-mode patterns of KIC 7694191. Panel a: the amplitude spectrum with x-axis of period. The y-axis is the amplitude in ppm. The solid red circles present the detected independent frequencies while the open red circles show the combination frequencies. The vertical dashed lines are the linear fits for each pattern. We found two independent frequency groups around $0.20 \mathrm{~d}$ and $0.38 \mathrm{~d}$. There are two period-spacing patterns. The blue one on the right is the $l=1, m=1 \mathrm{~g}$ modes while the cyan one on the left is the $l=2$, $m=2 \mathrm{~g}$ modes, whose periods are marked by the vertical dashed lines. Panel b: the period-spacing patterns of KIC 7694191. The linear fits and uncertainties are shown by the black and grey dashed lines with dips removed. The blue plus symbols are the dipole g modes and the cyan triangle symbols are the quadrupole g modes. Panels c and d: the detail of the spectrum and period-spacing pattern of quadrupole g modes. Panel e: the sideways échelle diagram of the quadrupole g-modes pattern. The cyan triangles are the periods belonging to the pattern while the black stars are the noisy peaks. Panel f: the normalised sideways échelle diagram of the quadrupole g-modes pattern. Panels g to $\mathrm{j}$ : same as $(\mathrm{c}-\mathrm{f})$ but for the dipole $\mathrm{g}$-mode patterns.

in Fig. 1a comprises the dipole $(l=1)$ sectoral $(m=l=1)$ g modes while the left one comprises the quadrupole $(l=2)$ sectoral $(m=l=2) \mathrm{g}$ modes. The mode identifications were based on the TAR fit and Saio et al. (2018b), as described below.

Fig. 1b presents the period spacing versus period. The period spacing for the dipole g modes decreases from $400 \mathrm{~s}$ to $100 \mathrm{~s}$ with increasing period. For the quadrupole g-mode pattern, the period spacing drops from $150 \mathrm{~s}$ to $50 \mathrm{~s}$ with increasing period. Both patterns show deviations from the linear model, such as the dip at $0.39 \mathrm{~d}$ in the dipole g-mode pattern. In a rapidly-rotating star, the dip is more likely 


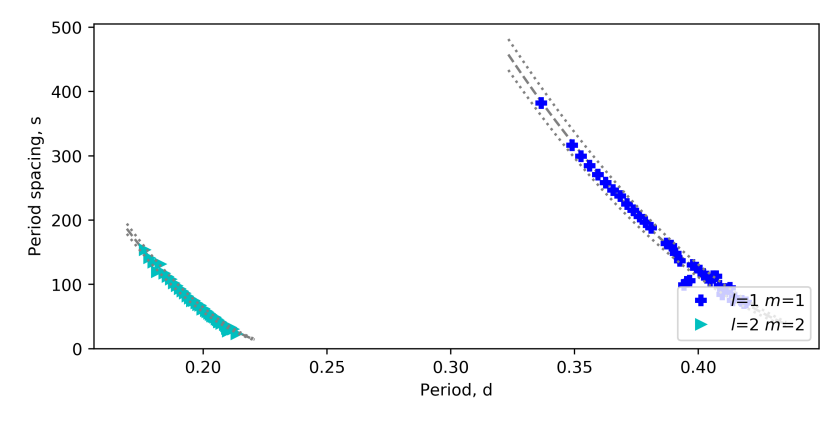

Figure 2. The TAR fitting of KIC 7694191. The dashed lines are the best-fitting result and the dotted lines show the uncertainty.

to form because of the mode coupling between sectoral and tesseral modes (Saio et al. 2018b). The linear fits and their uncertainties with the dips removed are shown by the black and grey dashed lines. Hence the linear fits are not affected by the dips.

After obtaining the initial estimates for the parameters from the cross-correlation algorithm, the sideways échelle diagram was made based on the formula

$P_{i}=\Delta P_{0} \frac{(1+\Sigma)^{i}-1}{\Sigma}+P_{0}=\Delta P_{0}\left(n^{\prime}+\epsilon\right)$,

with the assumption that the period spacing changes linearly with period. Here, $P_{i}$ is the $i^{\text {th }}$ pulsation period, $P_{0}$ is the first pulsation period, $\Delta P_{0}$ is the first period spacing, $\Sigma$ is the slope in the linear assumption, $n^{\prime} \equiv \frac{(1+\Sigma)^{i}-1}{\Sigma}$ is the normalised index, and $\epsilon$ is the ratio $P_{0} / \Delta P_{0}$ (Li et al. 2019a). The $\mathrm{x}$-axis of the sideways échelle diagram is the observed period and the $y$-axis is the difference between the observed and fitted periods from Eq. 1.

Panels (c) and (d) zoom in on the quadrupole g modes from panels (a) and (b), while panels (g) and (h) do the same for the dipole g modes. In Panels (e) and (i), the échelle diagrams are plotted sideways. The $\mathrm{x}$-axis is the pulsation period while the y-axis is the term $P^{\mathrm{obs}}-n^{\prime} \Delta P$ from the fit of eq. 1. During this fit, we did not exclude any dips. For the peaks that do not belong to the pattern, we plotted them at the location that minimised the value $P^{\mathrm{obs}}-n^{\prime} \Delta P$. Therefore, the y-axis reflects the deviation from the linear fit, similar to the curvature in the échelle diagram of solar-like oscillators (e.g. Mazumdar et al. 2014). In panel (e), the curve is smooth and is dominated by the slightly changing slope in the quadrupole sectoral g modes. In panel (i), there is a rapid drop at $0.39 \mathrm{~d}$, which is caused by the dip here. Panels (f) and (j) show the normalised sideways échelle diagram. The $\mathrm{x}$-axis is the index of peaks, counting the first peak as 0 , and the y-axis is the deviation over the local period spacing $\left(P^{\mathrm{obs}}-n^{\prime} \Delta P\right) / \Delta P$ expressed as a percentage.

For each period-spacing pattern, consisting of a series of pulsation periods $\left\{P_{i}\right\}$, we measured three observables: the mean period, the mean period spacing, and the slope. The mean period $\langle P\rangle$ is the average of the pulsation periods. The mean period spacing $\langle\Delta P\rangle$ is the slope of the linear fit between the periods $P_{i}$ and the index $i$. The slope $\Sigma$ is the changing rate between the period spacing and the period with dips removed.

After identifying a period-spacing pattern, the asymp- totic formulation of the Traditional Approximation of Rotation (TAR) was used to fit the pattern assuming rigid rotation (e.g. Eckart 1960; Lee \& Saio 1997; Townsend 2003a; Van Reeth et al. 2016). The pulsation periods in the corotating reference frame were computed by

$P_{n l m, \mathrm{co}}^{\mathrm{TAR}}=\frac{\Pi_{0}}{\sqrt{\lambda_{l, m, s}}}\left(n+\varepsilon_{g}\right)$,

where $\Pi_{0}$ is the asymptotic period spacing, $n$ is the radial order, the phase term $\varepsilon_{g}$ is set as 0.5 , and $\lambda_{l, m, s}$ is the eigenvalue of the Laplace tidal equation, which is specified by the angular degree $l$ for $\mathrm{g}$ modes or the value $k$ for $\mathrm{r}$ modes, the azimuthal order $m$ and the spin parameter $s$. The value $k$ is used since the angular degree $l$ is not defined for $\mathrm{r}$ modes (Lee \& Saio 1997). The spin parameter is defined as

$s \equiv \frac{2 f_{\mathrm{rot}}}{f_{\mathrm{co}}}$,

where $f_{\text {rot }}$ is the rotation frequency and $f_{\text {co }}$ is the pulsation frequency in the co-rotating frame. The TAR periods in the inertial reference frame are given by

$P_{n l m, \text { in }}^{\mathrm{TAR}}=\frac{1}{1 / P_{n l m, \mathrm{co}}^{\mathrm{TAR}}+m f_{\mathrm{rot}}}$.

Hence the near-core rotation rate, the asymptotic spacing, and the radial orders can be obtained by fitting these pulsation periods to the observed pattern using a Markov chain Monte Carlo (MCMC) optimising code described by $\mathrm{Li}$ et al. (2019b). Fig. 2 presents the TAR fitting result of KIC 7694191. The near-core rotation rate is $2.083 \pm$ $0.009 \mathrm{~d}^{-1}$ and the asymptotic spacing is $4400 \pm 200 \mathrm{~s}$. The bestfitting curves (dashed lines) follow the observed pattern and show slowly-changing slopes with period.

\section{RESULTS}

The parameters of the stars, the period-spacing patterns and the TAR fit results are listed in the online-only table, while table 1 shows part of the table for guidance on style and content. We also indicate which of the 97 stars have shortcadence data and we indicated which of the 124 stars show significant pressure modes oscillations. 10 have both. These give the possibility to investigate core-to-surface physics by using $\mathrm{g}$ and $\mathrm{p}$ modes together. All the period-spacing patterns are shown in Appendix A, which is also online-only. 
Table 1. KIC numbers, Kepler magnitudes $K_{p}$, effective temperatures $T_{\text {eff }}$, luminosities $L$, mode identifications (for g modes, we give the angular degrees $l$ and the azimuthal orders $m$, While for $\mathrm{r}$ modes, we list the value $k$ and $m$ ), mean pulsation periods $\langle P\rangle$, mean period spacings $\langle\Delta P\rangle$, slopes $\Sigma$, asymptotic spacings $\Pi_{0}$, near-core rotation rates $f_{\text {rot }}$, the ranges of radial orders $n$, and ranges of spin parameters $s$ of 611 stars in this paper. For the last two columns, ' 1 ' marks the stars which have short-cadence data (SC) or are $\gamma$ Dor- $\delta$ Sct hybrids (H).

\begin{tabular}{|c|c|c|c|c|c|c|c|c|c|c|c|c|c|c|c|c|c|}
\hline \multirow[t]{2}{*}{ KIC } & \multirow[t]{2}{*}{$K_{p}$} & \multirow{2}{*}{$\begin{array}{r}T_{\text {eff }} \\
\mathrm{K}\end{array}$} & \multirow{2}{*}{$\begin{array}{r}L \\
\mathrm{~L}_{\odot}\end{array}$} & \multirow[t]{2}{*}{$l$} & \multirow[t]{2}{*}{$k$} & \multirow[t]{2}{*}{$m$} & \multirow{2}{*}{$\begin{array}{r}\langle P\rangle \\
\text { days }\end{array}$} & \multirow{2}{*}{$\begin{array}{r}\langle\Delta P\rangle \\
\text { Seconds }\end{array}$} & \multirow{2}{*}{$\begin{array}{r}\Sigma \\
\text { days/days }\end{array}$} & \multirow{2}{*}{$\begin{array}{r}\Pi_{0} \\
\text { Seconds }\end{array}$} & \multirow{2}{*}{$\begin{array}{l}f_{\text {rot }} \\
\mathrm{d}^{-1}\end{array}$} & \multicolumn{2}{|c|}{$n$} & \multicolumn{2}{|c|}{$s$} & \multirow[t]{2}{*}{$\mathrm{SC}$} & \multirow[t]{2}{*}{$\mathrm{H}$} \\
\hline & & & & & & & & & & & & $\min$ & $\max$ & $\min$ & $\max$ & & \\
\hline \multirow[t]{2}{*}{1026861} & 11.00 & $7060 \pm 80$ & $8.5 \pm 0.5$ & 2 & & 2 & 0.8 & 530 & $-0.0123 \pm 0.0002$ & $4310 \pm 50$ & $0.311 \pm 0.002$ & 57 & 92 & 0.7 & 1.3 & 1 & \\
\hline & & & & 1 & & 1 & 1.5 & 1240 & $-0.0167 \pm 0.0007$ & & & 45 & 79 & 1.1 & 2.1 & & \\
\hline 1160891 & 13.21 & $6840 \pm 80$ & & 1 & & 1 & 0.6 & 200 & $-0.022 \pm 0.002$ & $2800 \pm 400$ & $1.14 \pm 0.03$ & 66 & 88 & 4.5 & 6.2 & & \\
\hline \multirow[t]{2}{*}{1162345} & 11.68 & $6500 \pm 200$ & $38 \pm 3$ & 2 & & 2 & 0.3 & 70 & $-0.0275 \pm 0.0002$ & $4400 \pm 400$ & $1.46 \pm 0.02$ & 55 & 71 & 3.9 & 5.1 & & \\
\hline & & & & 1 & & 1 & 0.5 & 290 & $-0.0372 \pm 0.0003$ & & & 35 & 47 & 4.8 & 6.6 & & \\
\hline 1295531 & 11.94 & $6760 \pm 80$ & & 1 & & 1 & 0.8 & 1280 & $-0.0266 \pm 0.0002$ & $4200 \pm 20$ & $0.572 \pm 0.002$ & 18 & 50 & 0.8 & 2.5 & & \\
\hline 1431379 & 12.62 & $6670 \pm 80$ & $8.5 \pm 0.7$ & 2 & & 2 & 0.3 & 200 & $-0.0384 \pm 0.0006$ & $4360 \pm 10$ & $1.2526 \pm 0.0006$ & 21 & 59 & 1.2 & 3.6 & & \\
\hline & & & & 1 & & 1 & 0.6 & 330 & $-0.0349 \pm 0.0005$ & & & 25 & 66 & 2.9 & 8.1 & & \\
\hline & & & & & -2 & -1 & 1.0 & 390 & $0.0504 \pm 0.0004$ & & & 12 & 60 & 7.1 & 23.1 & & \\
\hline 1432149 & 11.22 & $7500 \pm 300$ & $11.6 \pm 0.8$ & 2 & & 2 & 0.3 & 80 & $-0.0269 \pm 0.0003$ & $4200 \pm 400$ & $1.38 \pm 0.02$ & 54 & 75 & 3.4 & 4.8 & 1 & \\
\hline & & & & 1 & & 1 & 0.5 & 260 & $-0.0320 \pm 0.0004$ & & & 44 & 52 & 5.5 & 6.5 & & \\
\hline 1575977 & 13.61 & $7300 \pm 300$ & $8.8 \pm 0.7$ & 1 & & 1 & 0.4 & 710 & $-0.0543 \pm 0.0007$ & $3800 \pm 100$ & $1.50 \pm 0.02$ & 18 & 28 & 2.0 & 3.3 & & 1 \\
\hline 1872262 & 13.74 & $7100 \pm 200$ & $7.6 \pm 0.6$ & 2 & & 2 & 0.5 & 260 & $-0.028 \pm 0.002$ & $5000 \pm 2000$ & $0.70 \pm 0.05$ & 50 & 58 & 2.0 & 2.4 & & \\
\hline 1996456 & 11.44 & $7100 \pm 200$ & $10.1 \pm 0.8$ & 2 & & 2 & 0.4 & 160 & $-0.0294 \pm 0.0002$ & $4450 \pm 80$ & $1.038 \pm 0.004$ & 30 & 84 & 1.4 & 4.3 & 1 & \\
\hline & & & & 1 & & 1 & 0.7 & 420 & $-0.0338 \pm 0.0002$ & & & 30 & 63 & 2.8 & 6.3 & & \\
\hline 2018685 & 14.05 & $6700 \pm 200$ & $8.4 \pm 0.7$ & 2 & & 2 & 0.3 & 160 & $-0.0315 \pm 0.0004$ & $4200 \pm 200$ & $1.27 \pm 0.01$ & 32 & 57 & 1.8 & 3.4 & & \\
\hline & & & & 1 & & 1 & 0.6 & 350 & $-0.0343 \pm 0.0005$ & & & 35 & 50 & 4.1 & 5.9 & & \\
\hline 2020444 & 13.00 & $6890 \pm 80$ & $8.9 \pm 0.7$ & 1 & & 1 & 1.0 & 1040 & $-0.0211 \pm 0.0004$ & $4360 \pm 30$ & $0.499 \pm 0.002$ & 30 & 66 & 1.2 & 3.1 & & \\
\hline 2141387 & 12.15 & $7200 \pm 300$ & $7.0 \pm 0.6$ & 1 & & 1 & 1.0 & 1010 & $-0.035 \pm 0.001$ & $6090 \pm 90$ & $0.592 \pm 0.003$ & 29 & 51 & 2.1 & 4.0 & & \\
\hline 2163896 & 13.12 & $6900 \pm 200$ & $4.8 \pm 0.3$ & 1 & & 1 & 0.8 & 460 & $-0.0249 \pm 0.0002$ & $3400 \pm 100$ & $0.834 \pm 0.008$ & 44 & 74 & 2.5 & 4.5 & & \\
\hline 2168333 & 10.08 & $8400 \pm 300$ & $34 \pm 3$ & 1 & & 1 & 0.5 & 220 & $-0.0358 \pm 0.0004$ & $4200 \pm 200$ & $1.66 \pm 0.01$ & 31 & 62 & 4.5 & 9.5 & 1 & 1 \\
\hline 2300165 & 11.05 & $7400 \pm 300$ & $7.2 \pm 0.6$ & 2 & & 2 & 0.3 & 170 & $-0.0300 \pm 0.0002$ & $4140 \pm 70$ & $1.136 \pm 0.004$ & 29 & 75 & 1.4 & 3.9 & 1 & \\
\hline & & & & 1 & & 1 & 0.6 & 380 & $-0.0323 \pm 0.0003$ & & & 26 & 71 & 2.4 & 7.4 & & \\
\hline 2309579 & 13.35 & $7200 \pm 300$ & $10.0 \pm 0.7$ & 2 & & 2 & 0.4 & 110 & $-0.0211 \pm 0.0002$ & $4000 \pm 200$ & $1.001 \pm 0.007$ & 58 & 95 & 2.5 & 4.2 & & \\
\hline & & & & 1 & & 1 & 0.8 & 210 & $-0.0230 \pm 0.0001$ & & & 50 & 107 & 4.3 & 9.4 & & \\
\hline 2449383 & 13.92 & $7200 \pm 300$ & $7.6 \pm 0.6$ & 2 & & 2 & 0.2 & 90 & $-0.0310 \pm 0.0003$ & $3910 \pm 90$ & $1.588 \pm 0.007$ & 35 & 76 & 2.3 & 5.2 & & \\
\hline & & & & 1 & & 1 & 0.4 & 320 & $-0.0419 \pm 0.0004$ & & & 20 & 59 & 2.5 & 8.0 & & \\
\hline 2450944 & 15.74 & $6800 \pm 200$ & & 1 & & 1 & 1.0 & 2500 & $-0.0084 \pm 0.0004$ & $4136 \pm 5$ & $0.141 \pm 0.001$ & 24 & 37 & 0.2 & 0.3 & & 1 \\
\hline & & & & 1 & & -1 & 1.2 & 3350 & $0.003 \pm 0.001$ & & & 30 & 37 & 0.2 & 0.3 & & \\
\hline 2575161 & 10.88 & $6900 \pm 100$ & & 1 & & 1 & 0.4 & 250 & $-0.0466 \pm 0.0008$ & $4470 \pm 20$ & $1.833 \pm 0.001$ & 20 & 54 & 3.6 & 10.0 & & \\
\hline & & & & & -2 & -1 & 0.7 & 580 & $0.0753 \pm 0.0004$ & & & 8 & 27 & 7.3 & 16.2 & & \\
\hline 2578582 & 13.93 & $7300 \pm 300$ & $5.2 \pm 0.4$ & 1 & & 1 & 0.8 & 1140 & $-0.0233 \pm 0.0003$ & $3960 \pm 30$ & $0.570 \pm 0.003$ & 24 & 53 & 1.0 & 2.5 & & \\
\hline 2579147 & 13.83 & $7300 \pm 300$ & $14 \pm 1$ & 2 & & 2 & 0.7 & 620 & $-0.0096 \pm 0.0004$ & $3600 \pm 30$ & $0.286 \pm 0.003$ & 54 & 72 & 0.5 & 0.7 & & 1 \\
\hline & & & & 1 & & 1 & 1.1 & 1560 & $-0.0154 \pm 0.0006$ & & & 43 & 51 & 0.8 & 0.9 & & \\
\hline 2696217 & 13.45 & $7400 \pm 300$ & $7.0 \pm 0.5$ & 1 & & 1 & 1.3 & 1310 & $-0.0130 \pm 0.0002$ & $3460 \pm 20$ & $0.290 \pm 0.001$ & 39 & 84 & 0.7 & 1.7 & & \\
\hline 2710406 & 13.27 & $6960 \pm 80$ & $12.6 \pm 0.9$ & 1 & & 1 & 1.5 & 2270 & $-0.0054 \pm 0.0003$ & $4270 \pm 70$ & $0.138 \pm 0.006$ & 45 & 57 & 0.4 & 0.6 & & \\
\hline 2710594 & 11.79 & $7200 \pm 200$ & $8.0 \pm 0.5$ & 1 & & 1 & 0.7 & 360 & $-0.0290 \pm 0.0003$ & $4000 \pm 10$ & $0.9920 \pm 0.0006$ & 27 & 86 & 2.3 & 7.9 & & \\
\hline & & & & & -2 & -1 & 1.2 & 470 & $0.0381 \pm 0.0005$ & & & 20 & 64 & 7.4 & 18.0 & & \\
\hline 2719928 & 12.66 & $7200 \pm 200$ & $9.6 \pm 0.7$ & 2 & & 2 & 0.3 & 70 & $-0.0275 \pm 0.0004$ & $3700 \pm 700$ & $1.47 \pm 0.04$ & 55 & 82 & 3.3 & 5.0 & & \\
\hline & & & & 1 & & 1 & 0.5 & 150 & $-0.006 \pm 0.004$ & & & 59 & 69 & 7.1 & 8.4 & & \\
\hline 2846358 & 11.02 & $6800 \pm 200$ & & 2 & & 2 & 0.4 & 360 & $-0.0279 \pm 0.0004$ & $4020 \pm 50$ & $0.755 \pm 0.004$ & 25 & 61 & 0.7 & 2.0 & 1 & \\
\hline & & & & 1 & & 1 & 0.8 & 650 & $-0.0336 \pm 0.0004$ & & & 32 & 63 & 1.9 & 4.0 & & \\
\hline
\end{tabular}




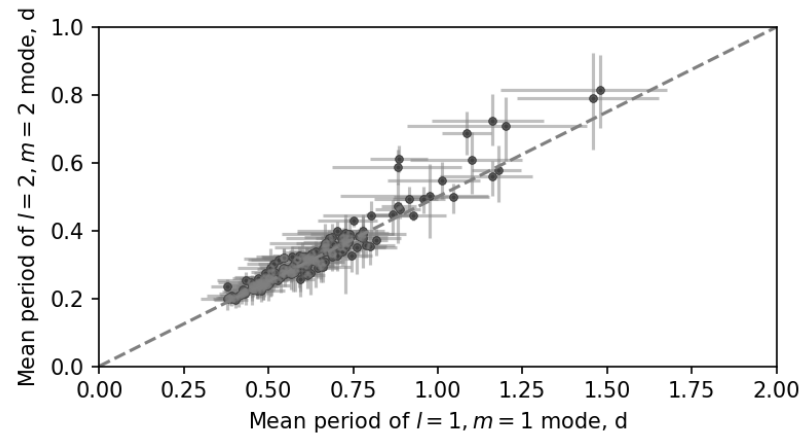

Figure 3. The observed period relation between $l=2, m=2$ $\mathrm{g}$ modes and $l=1, m=1 \mathrm{~g}$ modes. The error bars show the pulsation period spans, not the uncertainties. The dashed line shows the relation that the mean period of $l=2, m=2 \mathrm{~g}$ modes is half that of $l=1, m=1 \mathrm{~g}$ modes.

\subsection{Mode identification}

The periodogram of a $\gamma$ Dor star generally shows peak groups which overlap with the harmonics of fundamental frequencies. We accepted the explanation by Saio et al. (2018b) that the peak groups are prograde sectoral g-mode oscillations of increasing angular degree. Many quadrupole modes are seen in our sample. Figure 3 shows the correlation between the mean periods of $l=2, m=2$ and $l=1, m=1$ $\mathrm{g}$ modes. We find that the mean periods of quadrupole sectoral $\mathrm{g}$ modes are typically half those of dipole sectoral $\mathrm{g}$ modes, since the quadrupole sectoral g modes generally coincide with the second harmonics of dipole modes.

Figure 4 shows the slope relation between dipole and quadrupole g modes. We find that the slopes of $l=2, m=2$ g modes are similar but slightly smaller than those of $l=$ $1, m=1 \mathrm{~g}$ modes. We therefore conclude two features of the quadrupole sectoral g modes in $\gamma$ Dor stars:

- the mean period of the quadrupole modes is half that of the dipole sectoral $\mathrm{g}$ modes.

- the slopes of quadrupole and dipole sectoral $(l=m) \mathrm{g}$ modes are almost equal.

These features are common in most of stars and help identify the modes. If not, several conditions should be considered: if the power spectrum is contaminated by a binary, or if they are $m=1$ and $m=0$ modes showing large splittings (see the example and discussion in Section 6).

We plot the periodograms of all the $\gamma$ Dor stars in Fig. 5 Each row displays the normalised periodogram of one star, sorted vertically by the mean period of the dipole modes. Three ridges are seen: the dominant one is $l=1, m=1 \mathrm{~g}$ modes; the ridge of $l=2, m=2 \mathrm{~g}$ modes appears on the left, as these modes overlap with the second harmonics of the dipole modes; the third ridge is the $k=-2, m=-1 \mathrm{r}$ modes. We see that the $l=1, m=1 \mathrm{~g}$ modes in $\gamma$ Dor stars generally show the largest amplitudes. Assuming the dipole sectoral g modes appear around period of $P$, the quadrupole $g$ modes are expected to appear around $0.5 P$ and the $\mathrm{r}$ modes are more likely to appear around $2 P$ (Li et al. 2019b). This structure of $\gamma$ Dor periodograms helps guide the mode identification

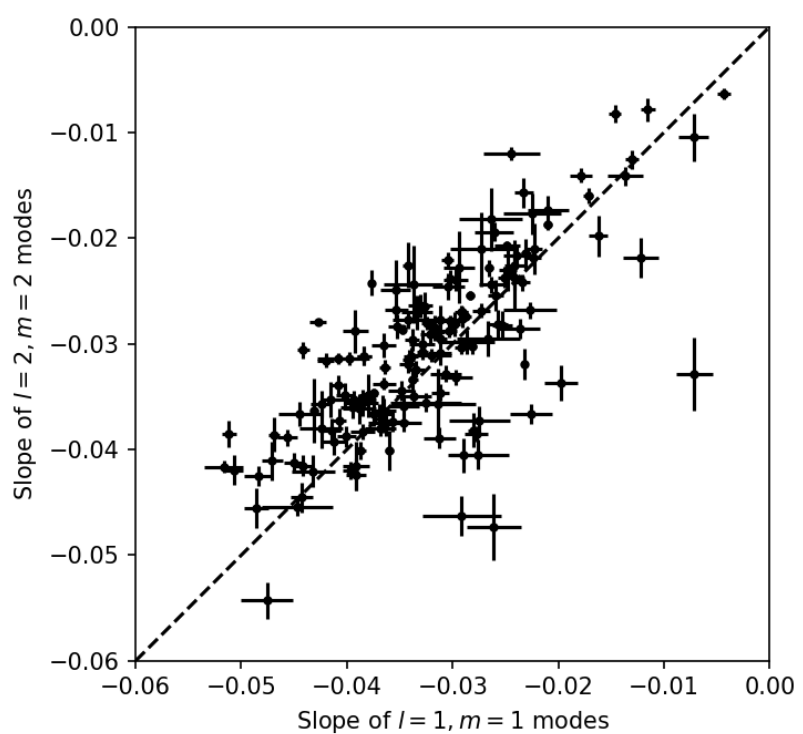

Figure 4. The slope relation between the observed $l=2, m=2$ and $l=1, m=1$ modes. We only plot the points with slope error smaller than 0.005. The dashed line shows the place where the slopes are equal.

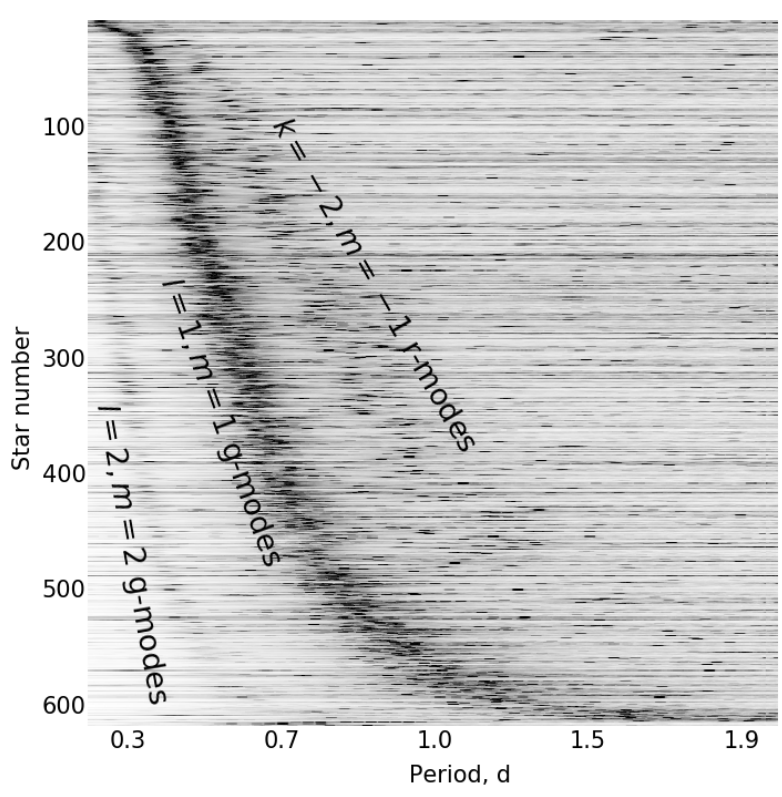

Figure 5. The periodograms of all the $\gamma$ Dor stars with identified period-spacing patterns in our sample. Each row shows the periodogram for one star. The colour stands for the normalised amplitudes to the power of 0.25 for the best visibility. The trends of $l=1, m=1 \mathrm{~g}$ modes, $l=2, m=2 \mathrm{~g}$ modes, and $k=-2, m=-1$ $\mathrm{r}$ modes are seen and marked by the texts. 




Figure 6. The percentages of different oscillation modes among 960 patterns.

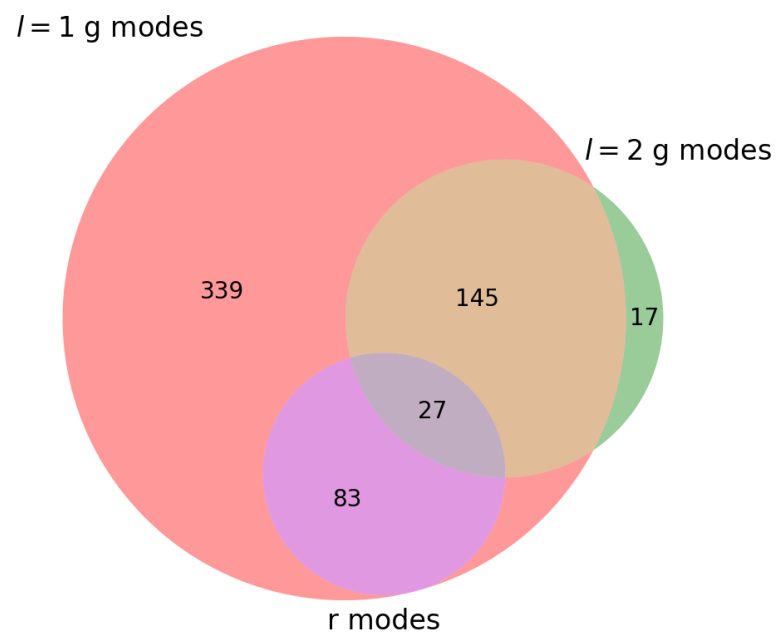

Figure 7. The numbers of stars with observed period-spacing patterns.

We find that four stars (KIC5876187, KIC9344493, KIC10091792, KIC10803371) show $l \geq 3$ g modes. These high $l \mathrm{~g}$ modes have smaller amplitudes and generally have periods below the lower boundary of our detection region $(0.2 \mathrm{~d}$ to $2 \mathrm{~d})$ hence they are hard to detect. However, for dipole and quadrupole g modes, we confirm that the results listed in Table 1 are complete.

\subsection{Occurence rate of modes}

Figure 6 shows the observed relative occurrence rates of different modes. Among all the 960 patterns, $62.0 \%$ are $l=1, m=1 \mathrm{~g}$ modes. The second most common modes are $l=2, m=2 \mathrm{~g}$ modes, which constitute $18.9 \%$ of the total detection. Rossby modes are the third most common modes (11.5\%). Apart from these three modes, we also see $l=1, m=0$ and $l=1, m=-1$ modes with percentages of $3.0 \%$ and $2.3 \%$, respectively, which were mainly found in the slow rotators reported by $\mathrm{Li}$ et al. (2019a). 11 fast rota- tors with splittings are detected in this work, which will be described in Section 6 .

There are a few patterns that cannot be classified into those five types of modes above. They might be the sectoral $\mathrm{g}$ modes with higher angular degree $(l=3, m=3$ for example, see KIC 9344493), or the only $k=-1, m=-1 \mathrm{r}$ mode reported by $\mathrm{Li}$ et al. (2019b), or $l=2, m=1 \mathrm{~g}$ modes in two newly-discovered fast rotators (KIC 5092681 and KIC 5544996), or some patterns that cannot be fitted with the TAR. All of these occupy $2.3 \%$ of the total detected period-spacing patterns.

Figure 7 shows the numbers of stars which show different oscillation modes. We classify the modes into three main types: $l=1 \mathrm{~g}$ modes, $l=2 \mathrm{~g}$ modes, and $k=-2, m=-1 \mathrm{r}$ modes. There are 339 stars that only show $l=1$ g modes (red area) and 145 stars that show both $l=1$ and $l=2 \mathrm{~g}$ modes (yellow area). Almost all the stars have $l=1$ g-mode period-spacing patterns. However, there are power excesses over $l=1 \mathrm{~g}$-modes regions in 16 stars without period-spacing patterns identified. For these stars only $l=2$ g-modes patterns are reported. We notice that KIC 5491390 is the only star that does not show any $l=1$ g-mode power excess. In total, there are 17 stars with only $l=2$ g modes (green area). Zhang et al. (2018) reported that KIC 10486425 also oscillates only in $l=2 \mathrm{~g}$ modes. The reason for the absence of $l=1 \mathrm{~g}$ modes needs further investigation.

We do not find any star that only shows $\mathrm{r}$ modes. The reason is that the TAR cannot converge well if g-mode patterns are absent, hence we cannot ensure that the observed pattern is a real $r$-mode pattern, or we are misled by missing peaks in the observed pulsation spectra. Hence, all the $\mathrm{r}$ modes co-exist with $\mathrm{g}$ modes in our sample. There are 83 stars with $l=1 \mathrm{~g}$ modes and $\mathrm{r}$ modes, and there are 27 stars with $l=1, l=2 \mathrm{~g}$ modes, and $\mathrm{r}$ modes. The co-existence of $g$ mode and $r$ modes decreases the uncertainties of nearcore rotation rates significantly. The typical uncertainty is $0.0009 \mathrm{~d}^{-1}$ for the stars with both $\mathrm{g}$ and $\mathrm{r}$ modes, while it is $0.008 \mathrm{~d}^{-1}$ for the stars with only $\mathrm{g}$ modes.

\subsection{Slope-Period diagram}

In Section 2, we introduced three observables for each pattern, the mean period, the mean period spacing, and the slope. Figure 8 shows the relation between the slopes and the mean periods from all the patterns in our sample, hence we call this diagram the Slope-Period (S-P) diagram. The mean period and the slope are correlated. We find that the data points of $l=1, m=1 \mathrm{~g}$ modes, $l=2, m=2 \mathrm{~g}$ modes, slowly-rotating $\mathrm{g}$ modes, and $k=-2, m=-1 \mathrm{r}$ modes form four different groups which have diverse trends and clear boundaries.

- $l=1, m=1 \mathrm{~g}$ modes: these points are the majority, which are shown by the blue triangles. Most patterns have mean periods between $0.4 \mathrm{~d}$ and $0.8 \mathrm{~d}$ and slopes around -0.04 . They show a positive correlation between the slopes and the mean periods.

- $l=2, m=2$ g modes: those points are presented by the green stars. These modes have shorter periods than dipole modes (between $0.2 \mathrm{~d}$ and $0.4 \mathrm{~d}$ ) but have similar slopes ( $-0.04)$ as pointed out in Section 3.1.

- $l=1, m=0$ and $l=1, m=-1 \mathrm{~g}$ modes: they are 


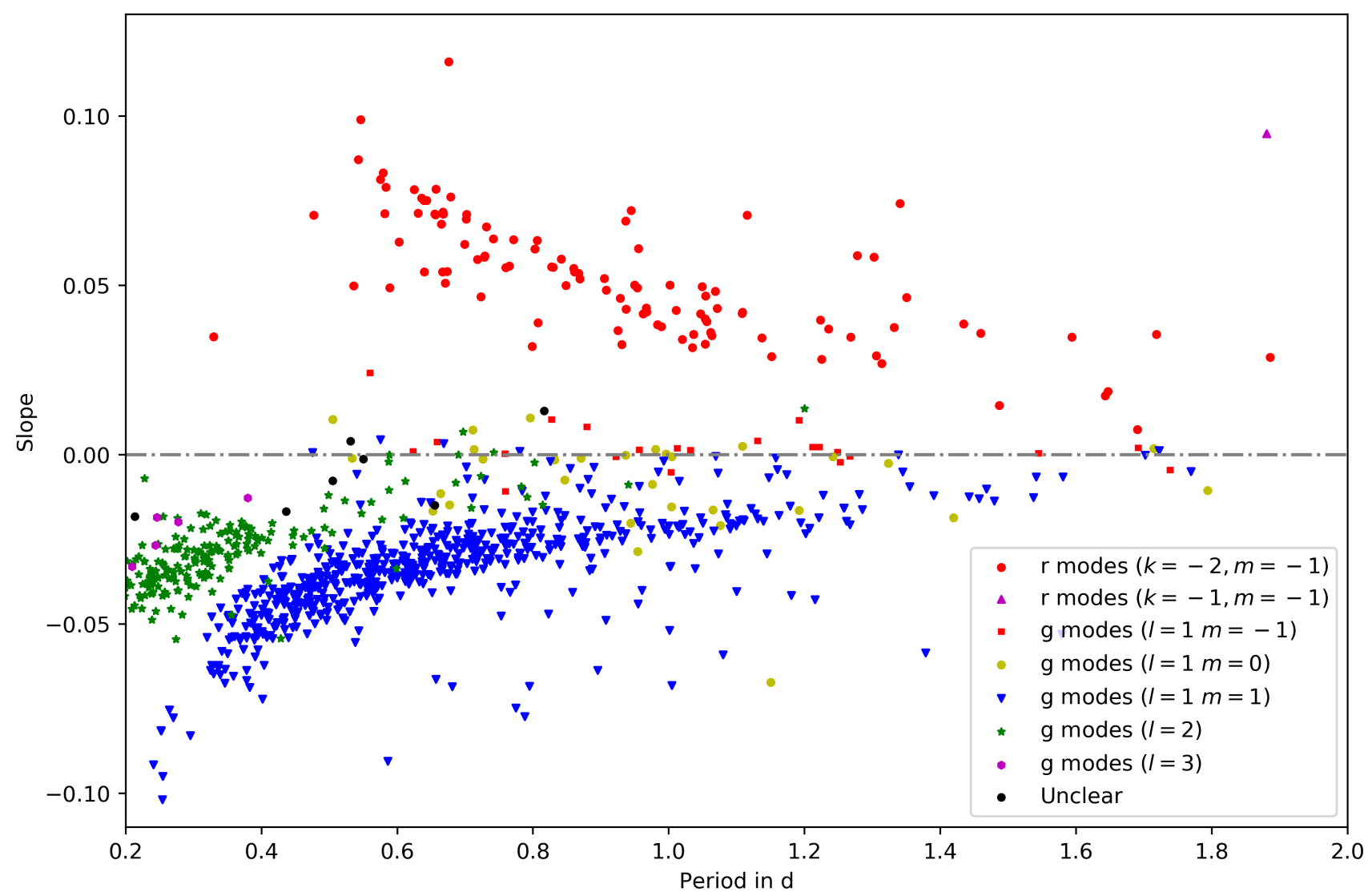

Figure 8. Slopes vs the means of the periods of 960 period-spacing patterns from $611 \gamma$ Dor stars. The $\mathrm{x}$-axis is the mean period in each pattern. The y-axis is the slope between the period spacing and the period with dips removed. Different colours and symbols show different modes.

marked by the yellow circles and the red rectangles. These two modes are rare (for $m=0$ ) or absent (for $m=-1$ ) in rapid rotators but are seen in the slow rotators reported by Li et al. (2019a). Due to the slow rotation rate, the rotational effect is not strong so the period spacings in those modes remain nearly identical. Hence we see most $m=0$ and $m=-1$ modes around the horizontal line with slope of zero.

- $k=-2, m=-1 \mathrm{r}$ modes: they are the red circles. As discussed by Li et al. (2019b), r modes have positive slopes and show an inverse correlation between the mean period and the slope.

Figure 8 displays the typical locations of different modes on the S-P diagram. It can be used for mode identification. When a new pattern is found, its location on the $\mathrm{S}-\mathrm{P}$ diagram reveals its mode identification. If the point is an outlier, several possibilities should be considered: the period spacings are misidentified since some peaks in the amplitude spectra are too weak to be detected; the slope is strongly affected by the partially-observed dips caused by chemical composition gradients (e.g. KIC 4919344 in Li et al. 2019a); or the star is a Slowly Pulsating B (SPB) star, which has a larger asymptotic spacing $\left(\Pi_{0}\right)$ because it has a higher mass than a $\gamma$ Dor star (e.g. Pápics et al. 2017). Consequently, a pattern of an SPB star has a steeper slope than a pattern of a $\gamma$ Dor star with a similar mean period.

\section{ASYMPTOTIC SPACING AND ROTATION}

We used the TAR to fit the period-spacing patterns and measured the near-core rotation rates $f_{\text {rot }}$, the asymptotic spacings $\Pi_{0}$ (also called buoyancy radii), and the radial orders $n$.

\subsection{Distribution of $\Pi_{0}$}

Figure 9 gives the observed distributions of the asymptotic spacing $\Pi_{0}$. The stars show a symmetric distribution centred around $\Pi_{0}=4000 \mathrm{~s}$. We find that $68 \%$ of stars have $\Pi_{0}$ between $3700 \mathrm{~s}$ and $4800 \mathrm{~s}$. Stars with large $\Pi_{0}$ are likely to be Slowly Pulsating B (SPB) stars (with $\Pi_{0}$ from $5600 \mathrm{~s}$ to 16000 s Pápics et al. 2017). They show g-mode patterns with larger period-spacing values and steeper slopes than $\gamma$ Dor stars but the pulsation periods are similar. However, the effective temperatures of those possible SPB stars are located in the typical ranges of A- and F-type stars. This may indicate that the effective temperatures are wrong, or there are pulsation periods missing in the detected patterns, or the stars are very young. The stars with $\Pi_{0} \lesssim 3000$ s are probably close to the terminal age main sequence.

Van Reeth et al. (2016) reported the theoretical distribution of $\Pi_{0}$, which was calculated based on a grid of 


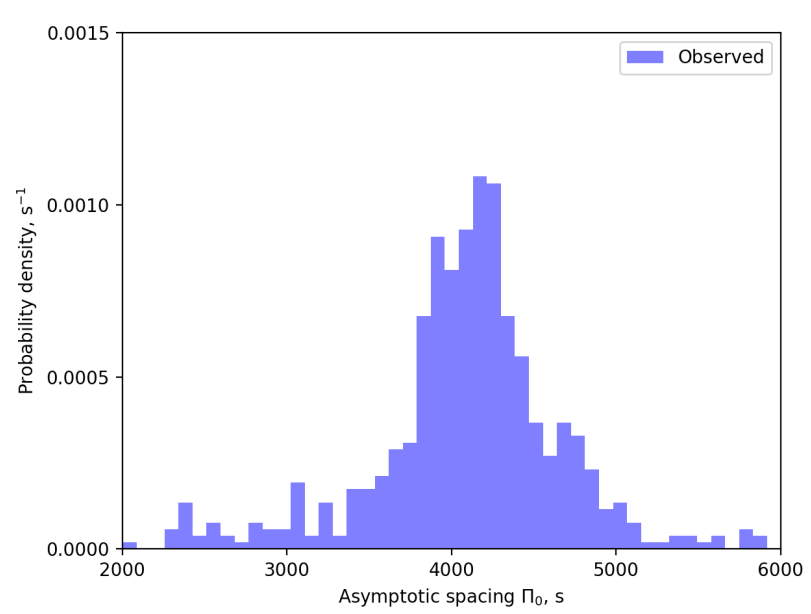

Figure 9. The observed distribution of asymptotic spacing $\Pi_{0}$.

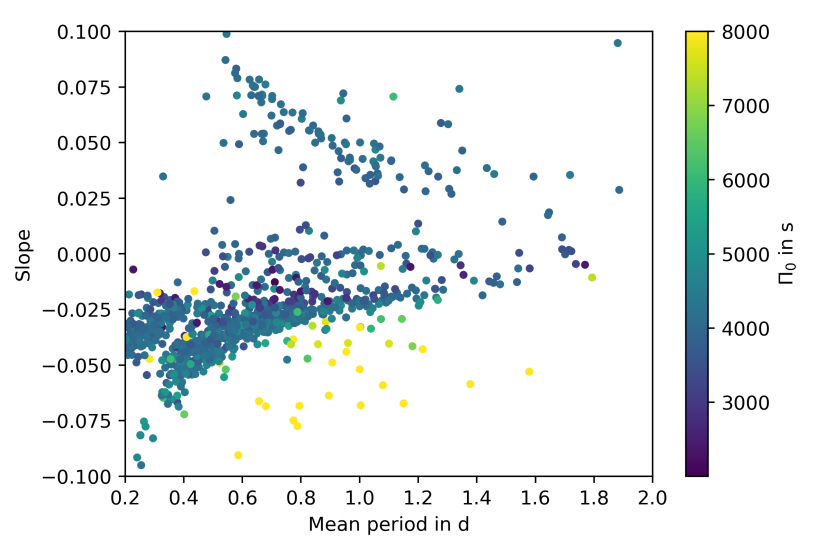

Figure 10. The S-P diagram coloured by the asymptotic spacing $\Pi_{0}$. The outliers on the lower right is composed of the stars with large $\Pi_{0}$.

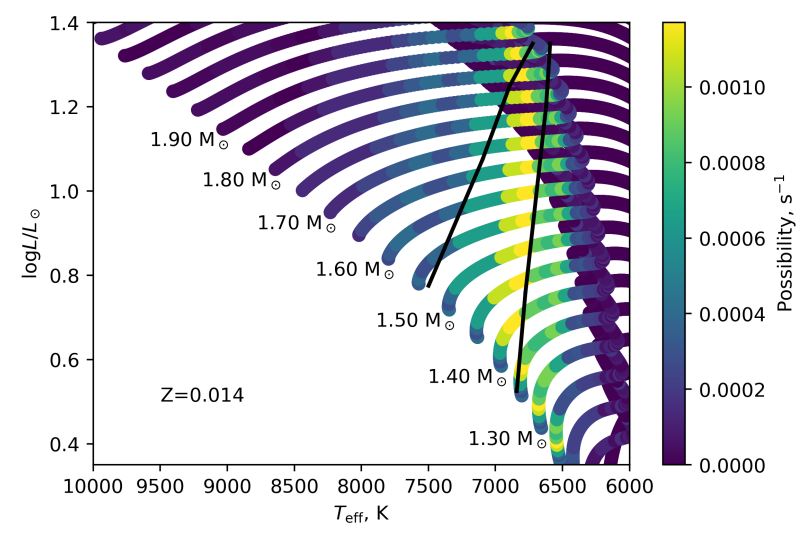

Figure 11. The probability density of $\Pi_{0}$ on the HR diagram. The colour stands for the probability density of $\Pi_{0}$ from Fig. 9 . The black lines show the theoretical instability strip of $\gamma$ Dor stars (Dupret et al. 2005).

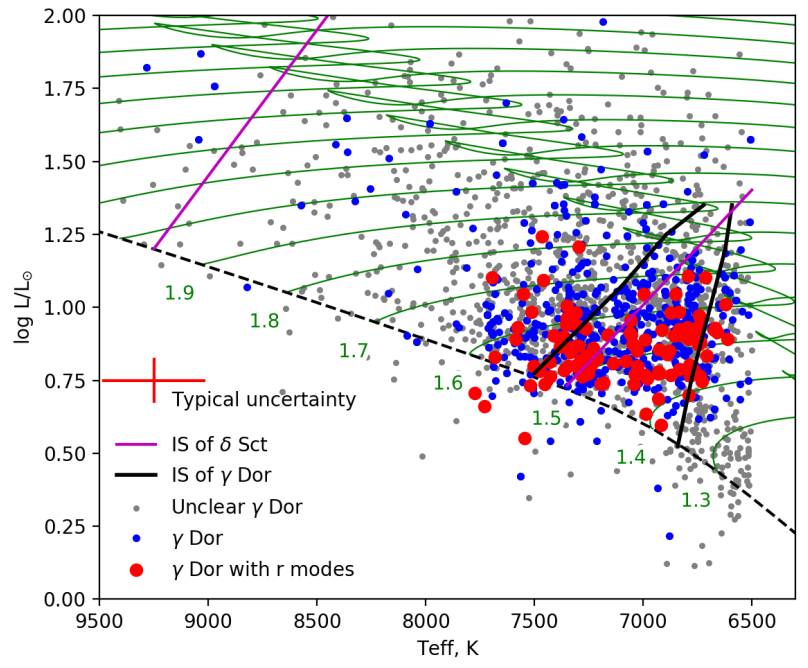

Figure 12. Stars on the HR diagram. The blue points are the $\gamma$ Dor stars with clear g-mode patterns. The red points are the stars with both g- and r-mode patterns. The grey points are the stars we inspected but do not show clear period spacing patterns. The black lines show the theoretical IS of $\gamma$ Dor star while the purple lines depict the observed IS of $\delta$ Sct star by Murphy et al. (2019).

theoretical stellar models that includes the $\gamma$ Dor instability strip. The relative duration of the different evolutionary stages was also considered in the calculation. It shows that the most likely value of $\Pi_{0}$ is $4400 \mathrm{~s}$, which is higher than the observed one. The theoretical histogram also has a slightly asymmetric shape. The discrepancy between the observed and theoretical distributions are probably caused by the different parameters, such as metallicity and mixing length, and it also reveals that a full non-adiabatic computation of the $\gamma$ Dor instability strip is needed for the theoretical distribution of $\Pi_{0}$.

Figure 10 shows the S-P diagram coloured by their asymptotic spacings $\Pi_{0}$. We found that the yellow outliers on the lower right are composed of the stars with large $\Pi_{0}$. They generally show steeper period-spacing patterns hence they appear below the typical $l=1, m=1$ g-mode group of $\gamma$ Dor stars. From now, we only present the results using stars with $\Pi_{0}<6000$ s to avoid any contamination from SPB stars or wrong identifications.

We show our theoretical evolutionary tracks in Fig. 11. MESA v10108 was used to compute the evolutionary tracks (Paxton et al. 2011, 2013, 2015, 2018). The tracks shown in Fig. 11 have: stellar masses are from 1.0 to $3.0 \mathrm{M}_{\odot}$ with step of $0.05 \mathrm{M}_{\odot}$, a hydrogen mass fraction $X$ of 0.71 , a metallicity $Z$ of 0.014 , a mixing length $\alpha$ of 1.8 , an exponential core overshooting $f_{\mathrm{ov}}$ of 0.015 , and an extra diffusive mixing $D_{\text {mix }}$ of $1 \mathrm{~cm}^{2} \mathrm{~s}^{-1}$, we also used the OPAL capacities and the Asplund et al. (2009) solar abundance mixture. For each stellar model, the asymptotic spacing $\Pi_{0}$ is calculated and the point is coloured by the observed probability density of $\Pi_{0}$ from Fig. 9. Two solid black lines in Fig. 11 display the boundaries of the theoretical instability strip of $\gamma$ Dor stars (Dupret et al. 2005). We find that the areas with high $\Pi_{0}$ densities show a nearly-vertical strip, broad at the ZAMS 
and narrow at the TAMS. The low-mass stars are more likely to pulsate near the ZAMS, while for the high-mass stars, the pulsation may happen close to the TAMS and for a shorter duration than for low-mass stars. The high-density area of $\Pi_{0}$ on the HR diagram is generally consistent with the theoretical instability strip.

Combining the effective temperatures from Kepler DR25 (Mathur et al. 2017), and the luminosities from Murphy et al. (2019) using gaia DR2 parallax (Gaia Collaboration et al. 2016), we place our stars on the HR diagram, as shown in Fig. 12. Figure 12 displays that most $\gamma$ Dor stars are located on the lower right area, with lower effective temperature and luminosity than $\delta$ Sct stars. The lowtemperature boundary of our $\gamma$ Dor sample follows the theoretical instability strip (solid black lines). This may prove that the theory predicted the red boundary correctly. However, many $\gamma$ Dor stars are located beyond the blue boundary of the instability strip. This could be caused by systematic offsets in the photometric $T_{\text {eff }}$ values. Typical uncertainties on these values are on the order of $250 \mathrm{~K}$. More accurate $T_{\text {eff }}$ values from high-resolution spectroscopy are needed to evaluate this possibility. If the $T_{\mathrm{eff}}$ values are found to be accurate, the presence of hot $\gamma$ Dor stars could reflect the limit of the current theory, which was mentioned by Dupret et al. (2005). For example, a proper mixing length should be used in these stars rather than the solar value.

As mentioned before, we inspected 2085 stars and found 611 stars with clear period-spacing patterns. The grey circles in Fig. 12 are the stars without identified period-spacing patterns. They may be the $\gamma$ Dor stars with unresolved gmode patterns, or the phase-modulation binaries from Murphy et al. (2018). We included the phase-modulation binaries since they show similar $T_{\text {eff }}$, but they may not necessarily be $\gamma$ Dor stars. We do not find any special distributions of the stars without pulsation patterns on the HR diagram. There is no explanation about why some $\gamma$ Dor stars do not show any clear period-spacing pattern. The reasons might be: dense, overlapping patterns that would be hard to disentangle; there are only a few excited modes hence the pattern is incomplete.

\subsection{Distribution of $f_{\text {rot }}$ with slow-rotator excess}

Figure 13 displays the distribution of the near-core rotation rates $f_{\text {rot }}$. Most stars have rotation frequencies around $1 \mathrm{~d}^{-1}$. The distribution increases rapidly after $0.4 \mathrm{~d}^{-1}$ and drops slowly after $\sim 1 \mathrm{~d}^{-1}$. The most rapid rotators are KIC 8458690A and KIC 8458690B with $f_{\text {rot }} \sim 3.01 \mathrm{~d}^{-1}$, whose two identical period-spacing patterns form 'splittings' reported by Li et al. (2019a). However, many stars rotate less quickly than expected, which forms an excess at $f_{\text {rot }}$ $\lesssim 0.4 \mathrm{~d}^{-1}$ in Fig. 13 .

The histogram of the near-core rotation rate in Fig. 13 shows a slow-rotator excess. We suggest defining two classes of $\gamma$ Dor stars by their near-core rotation rates: (1) slow rotators with $f_{\text {rot }} \lesssim 0.4 \mathrm{~d}^{-1} ;(2)$ fast rotators with $f_{\text {rot }} \gtrsim 0.4 \mathrm{~d}^{-1}$. A similar distribution has been realised for A- and F-type stars by observing the projected velocity $v \sin i$ (e.g. Ramella et al. 1989; Abt \& Morrell 1995; Royer et al. 2007). Abt \& Morrell (1995) found that all the rapid rotators have normal spectra and nearly all slow rotators have abnormal spectra (Ap or Am). The extremely slow rotation rate may be ex-



Figure 13. The distribution of observed near-core rotation rate $f_{\text {rot }}$. Many stars rotate around $1 \mathrm{~d}^{-1}$ while there is a slow-rotator excess slower than $0.4 \mathrm{~d}^{-1}$, suggesting two classes of $\gamma$ Dor stars.

plained by magnetic braking for Ap stars, or tidal braking for Am stars. However, after removing Ap and Am stars, Royer et al. (2007) still found the bimodality. The slow rotators have $v \sin i<70 \mathrm{~km} / \mathrm{s}$ and the fast rotators have $v \sin i \sim 160 \mathrm{~km} / \mathrm{s}$, whose ratio is consistent with our nearcore rotation rate $\left(0.4 \mathrm{~d}^{-1}\right.$ and $\left.1.0 \mathrm{~d}^{-1}\right)$. Due to the large sample size here, the effect of inclination should be averaged out hence we compare $v \sin i$ with our inclination-independent near-core rotation rate in the last sentence directly. Rotational braking during the main sequence can be explained in many ways, such as magnetic fields, binarity, interaction with stellar disc, or the formation of blue stragglers (e.g. Mestel 1968; Hut 1981; Takada-Hidai et al. 2017). Our sample contains a large number of slow rotators. Follow-up spectroscopic observations can obtain the chemical abundances and the surface rotations, hence we can infer the formation of the slow rotators.

According to Ouazzani et al. (2017), the slope $\Sigma=$ $\mathrm{d} \Delta P / \mathrm{d} P$ was defined as a diagnostic for rotation. The slope decreases from zero with increasing rotation for $m \geq 0 \mathrm{~g}$ modes and vice versa for the r-mode pattern. We plot the relation between the fitted near-core rotation rate and the observed slope in Fig. 14. The points clustered into two groups, corresponding to $l=1, m-1 \mathrm{~g}$ modes and $k=-2, m=-1$ $r$ modes. For the g-mode patterns, only several slow rotators show g-mode slopes slightly larger than 0 and most points have negative slopes and are located on the left side of Fig. 14. We find the rotation-slope relation of the g modes has a large scatter $\left(\sigma=0.35 \mathrm{~d}^{-1}\right)$ and shows an obvious gradient with the mean radial orders. The gradient reveals that the slope for a period-spacing pattern is not only affected by the rotations and dips, but also affected by the radial orders. For a given rotation rate (for example $1 \mathrm{~d}^{-1}$ ), the slopes are generally flatter ( $\Sigma$ near zero) for higher radial orders. The effect of radial orders is clear and can be used to explain the widths of the trends in Fig. 8. Further discussion about the radial orders on the $\mathrm{S}-\mathrm{P}$ diagram will be given in Section 5 and Fig. 21.

For the $\mathrm{r}$ modes, the rotation rate has a positive correlation with the slope. There is less scatter among the $\mathrm{r}-$ mode points $\left(\sigma=0.24 \mathrm{~d}^{-1}\right)$, presumably because they show 


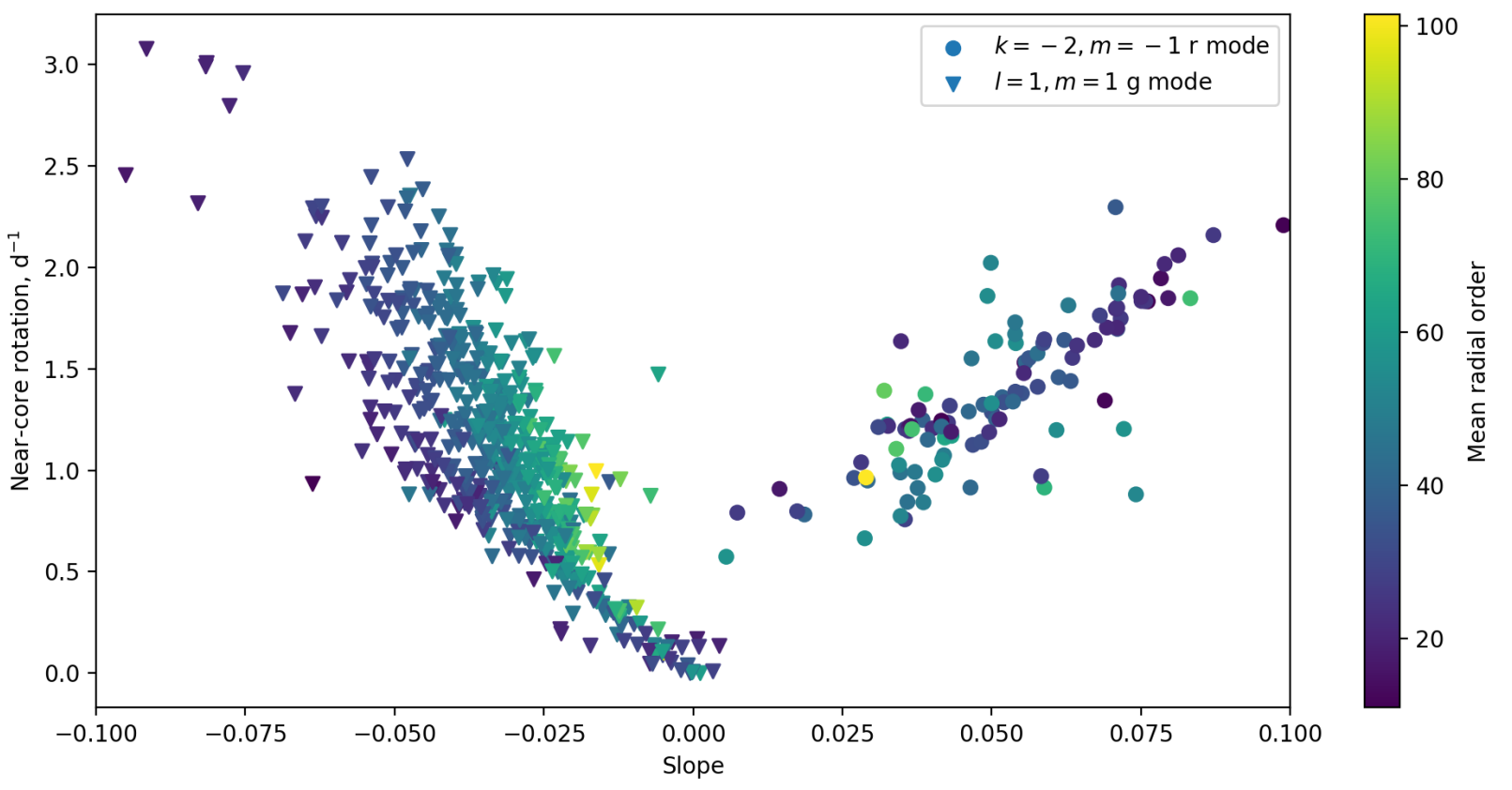

Figure 14. The relation between slope and near-core rotation rate $f_{\text {rot }}$. The triangles are the $l=1, m=1 \mathrm{~g}$-mode patterns, whose slopes are generally smaller than zero hence are located on the left. The circles are the $k=-2, m=-1 \mathrm{r}$-mode patterns with positive slopes on the right. The g-mode slopes are correlated with their mean radial orders, as shown by the colour gradient.

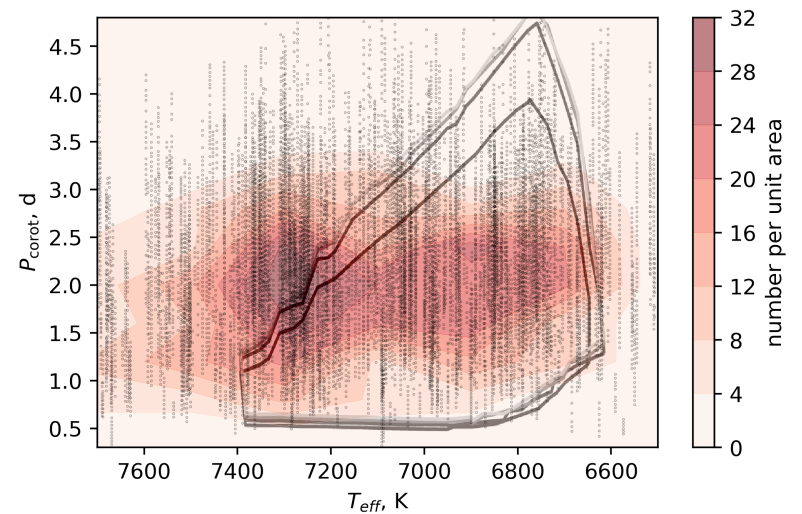

Figure 15. Pulsation period in the co-rotating frame vs effective temperature. One star has one effective temperature but many pulsation modes hence the data points (black dots) show vertical fringes. The contour displays the number of the observed pulsation modes. The solid lines show the theoretical areas reported by Bouabid et al. (2013), with equatorial rotation velocities $v_{\mathrm{eq}}=0$ (black), 30 (dark grey), 60 (grey) and $90 \mathrm{~km} / \mathrm{s}$ (light grey).

a smaller spread in radial orders, as we investigate in Section 4.4. The theoretical relation between slope and nearcore rotation rate also depends on the stellar parameters (such as $T_{\text {eff }},[\mathrm{Fe} / \mathrm{H}]$ ), which should be considered when comparing with observations.

Bouabid et al. (2013) predicted the relation between the pulsation period in the co-rotating frame vs the effective temperature, shown as the solid lines in Fig. 15. It pre- dicted that $\gamma$ Dor stars pulsate between 0.5 and $5 \mathrm{~d}$ in the co-rotating frame with effective temperature from 6600 to $7400 \mathrm{~K}$. The area is triangular, implying that the long-period stars are more likely to have lower temperatures. We count the number of the $l=1, m=1 \mathrm{~g}$ modes and compare our observations with the theoretical prediction in Fig. 15. The pulsation period in the inertial frame is converted into the co-rotating frame using the near-core rotation rate derived by the TAR fit. It shows that the co-rotating periods are generally between 1 and $4 \mathrm{~d}$, following the theoretical prediction. However, many stars have higher photometric temperatures than the theory, which is similar to what the H-R diagram shows in Fig. 12. The peak of the observed contour is located outside the theoretical area and the pulsation period does not show any relation with effective temperature.

\subsection{Correlation between $\Pi_{0}$ and $f_{\text {rot }}$}

Figure 16 shows the correlation between the near-core rotation rate and the asymptotic spacing. The uncertainty on $\Pi_{0}$ is sometimes large, due to short patterns, or when only $l=2, m=2 \mathrm{~g}$ modes are seen. Hence, we only plot the 578 stars with $\Pi_{0}$ uncertainty within \pm 500 s. The asymptotic spacing decreases with stellar evolution so it is considered an indicator of the stellar age (e.g. Saio et al. 2015; Ouazzani et al. 2018). However, the relation between $\Pi_{0}$ and age is affected by many other issues, for example, the shape of the instability strip (Fig. 11), or the initial mass. A detailed relation was reported by Mombarg et al. (2019). With stellar evolution, angular momentum is transferred and the nearcore rotation also decreases. Hence, in Figure 16, the stars evolve from upper-right to lower-left. 


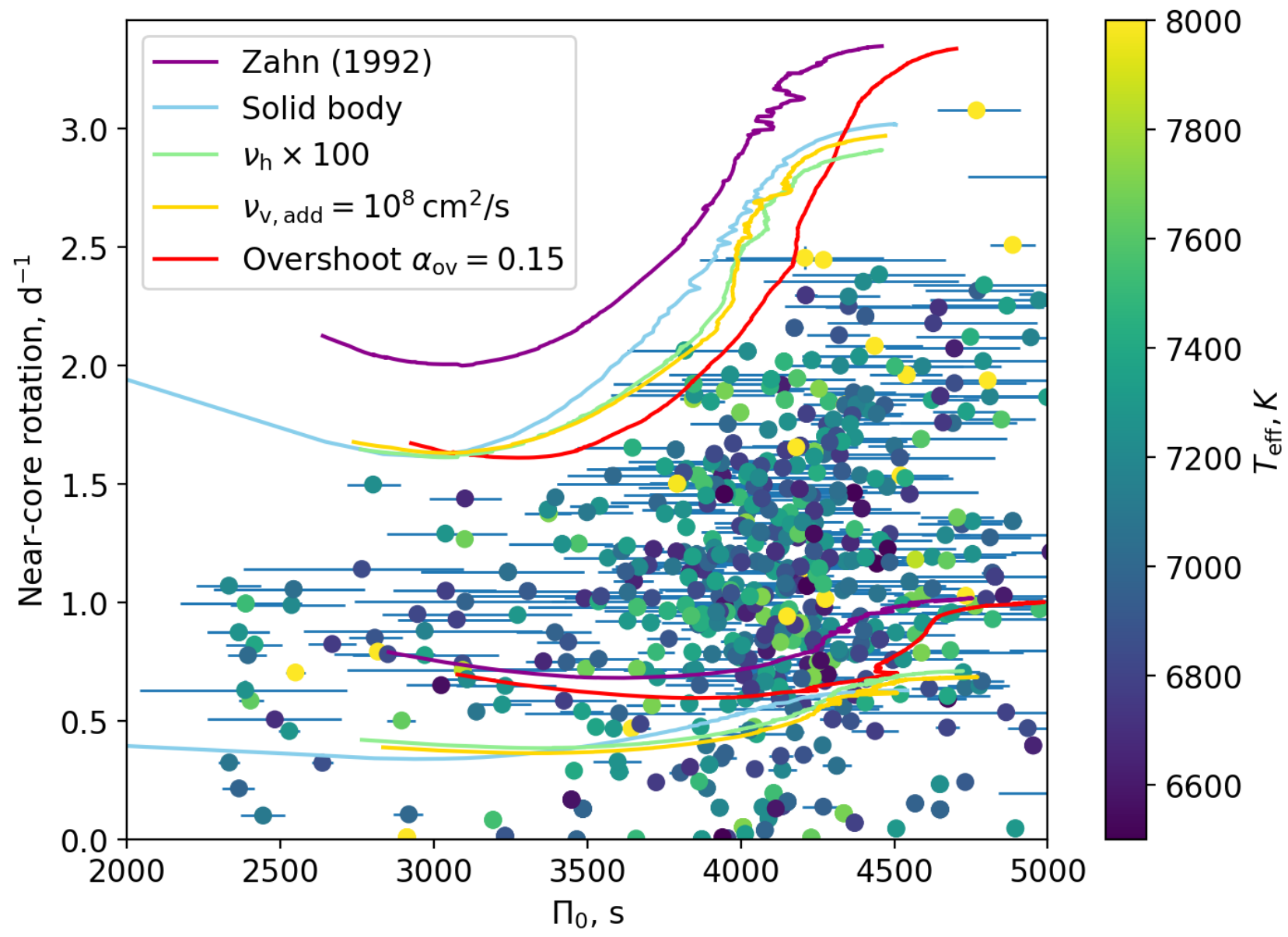

Figure 16. The correlation between asymptotic spacing $\Pi_{0}$ and the near-core rotation rate $f_{\text {rot }}$. With stellar evolution, the star moves from right to left. We only plot the points with $\Pi_{0}$ uncertainty smaller than $500 \mathrm{~s}$. The colour stands for the effective temperature. The theoretical predictions (solid lines) are made by Zahn (1992) and Ouazzani et al. (2018), where $v_{\mathrm{h}}$ means the enhanced horizontal viscosity, $v_{\mathrm{v}, \text { add }}$ means the additional vertical viscosity.

The solid lines show the theoretical boundaries calculated by the angular momentum transfer model of Zahn (1992), which considered the effects of meridional circulation and shear-induced turbulence and were calibrated by the observations of three clusters (Ouazzani et al. 2018). We plot the theoretical boundaries with different conditions, such as the original model by Zahn (1992) (purple lines), the model assuming stars are solid bodies (blue lines), the Zahn (1992) model with enhanced horizontal viscosity $v_{\mathrm{h}} \times 100$ (green lines), or with additional vertical viscosity $v_{\mathrm{v}, \text { add }}$ (yellow lines), or with overshooting $\alpha_{\mathrm{ov}}=0.15$ (red lines) (see details in Ouazzani et al. 2018).

Our observational points are generally located between the theoretical lines. The upper boundaries of the models from Ouazzani et al. (2018) fit the observations very well, which were calibrated by three clusters to include $80 \%$ of the stars. Our observations do not have any star above these upper boundaries, implying that there is a lack of fast rotators. We also notice that there are still many slow rotators below the lower boundaries of these models, confirming the 'slow rotator accumulation' by Ouazzani et al. (2018). Our results are consistent with and expand upon the results from 37 stars by Ouazzani et al. (2018) (these stars are also in our sample).

The difference between the observations and the theory demands an explanation. Either there is a selection effect in the observations, or there are ingredients missing from the stellar models that produce the theoretical predictions. We consider the former, first.

The 'fast rotators desert' might be expected if the period spacing patterns of rapid rotators cannot be extracted from (evolved) stars with small asymptotic spacings, as is indeed the case. In other words, although patterns are extractable for $\Pi_{0}=5000 \mathrm{~s}$ and $f_{\text {rot }}=2.5 \mathrm{~d}^{-1}$, they are not extractable for $\Pi_{0}=3000 \mathrm{~s}$ at the same $f_{\text {rot }}$ due to a denser power spectrum. However, the shape of the theoretical regions in Fig. 16 matches the observational distribution and is only shifted from it. Stars are not predicted at $\Pi_{0}=3000 \mathrm{~s}$ and $f_{\text {rot }}=2.5 \mathrm{~d}^{-1}$, so the 'fast rotators desert' is not the result of an observational selection effect.

What ingredients might be missing from the models that would move the theoretical region towards the observed 


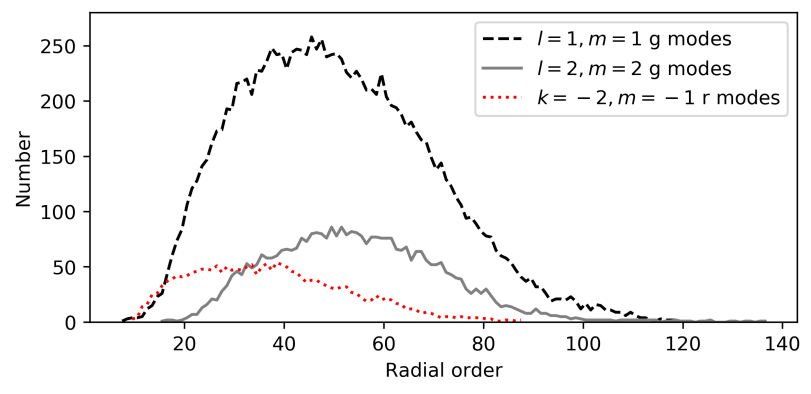

Figure 17. The distributions of radial orders of different modes.

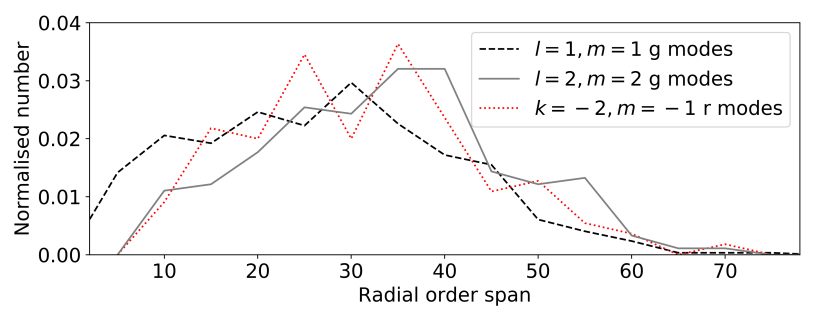

Figure 18. The normalised distributions of the pattern lengths.

one? One possible answer is the rigid rotation. As pointed out by Van Reeth et al. (2018); Li et al. (2019b) and discussed in Section 7 , the $\gamma$ Dor stars have almost the same rotation rates between near-core and surface regions, implying a very effective mechanism of angular momentum transfer. The model with solid body condition (light blue lines in Fig. 16) indeed shifts down and is a better match to the observations. Ouazzani et al. (2018) also modified different coefficients beyond their ordinary range to investigate the effect of the models, such as enhancing horizontal viscosity in the star by a factor 100 , which also have the desired effects. Another governing variable is the asymptotic spacings (or called 'buoyancy radius' in Ouazzani et al. 2018); increasing the asymptotic spacings moves the theoretical region down in Fig. 16. An additional parameter that modifies the asymptotic spacings is convective overshooting above the core. Including this parameter is physically motivated, it migrates the theoretical boundaries in the direction of the observations, and may fully resolve the difference between the theory and observations, as what we see in Fig. 16.

\subsection{Distributions of radial orders}

Figure 17 depicts the distributions of the radial orders for different modes, which are obtained by the best-fitting results of the TAR. We find that the distributions of the radial orders are similar to the results by $\mathrm{Li}$ et al. (2019b). For $l=1, m=1 \mathrm{~g}$ modes, the median of the distribution is 48 , and $68 \%$ of modes have radial orders between 30 and 70 . For $l=2, m=2 \mathrm{~g}$ modes, the peak of the distribution has a slightly higher radial order than dipole g modes, and $68 \%$ of modes satisfy $37<n<71$. We notice that the radial orders are higher than the theoretical prediction by Bouabid et al.

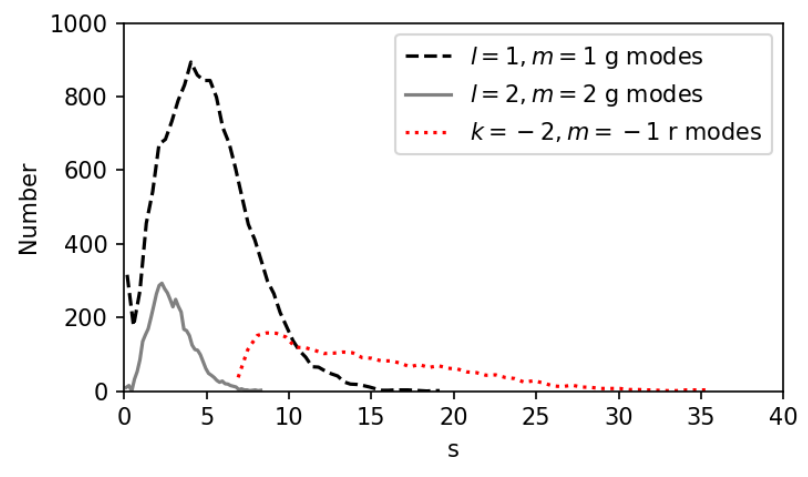

Figure 19. The spin parameter $s$ distributions of $l=1, m=1 \mathrm{~g}$ modes (black), $l=2, \quad m=2 \mathrm{~g}$ modes (grey), and $k=-2, m=-1$ $\mathrm{r}$ modes (red). Several extremely slowly rotating stars contribute the peak with $s$ near 0 in $l=1, m=1 \mathrm{~g}$ modes.

(2013), which found that the modes with radial orders from 15 to 38 are unstable.

For $k=-2, m=-1 \mathrm{r}$ modes, the radial orders are generally lower than those of $g$ modes. The median is 36 , and $21<n<53$ is the range for $68 \%$ of the modes. The distribution of $\mathrm{r}$-mode radial orders is asymmetric while the distributions of $\mathrm{g}$ modes are almost symmetric.

Figure 18 presents the pattern lengths for different modes. The pattern length is the difference between the maximum and minimum of the radial orders. The numbers are normalised for a clear comparison. We find that the lengths for dipole $g$ modes, quadrupole g modes, and $r$ modes do not show any dramatic differences. The medians are about 30 and most of them have pattern lengths between 10 and 50 radial orders. Several patterns are extremely long, even up to 70 radial orders.

Bouabid et al. (2013) calculated the radial order span using the theory of mode stability and found the radial order span is typically 30 . Our observed radial order span is longer than the theory, showing that improvement of the mode excitation and damping theory may be needed.

\subsection{Distributions of spin parameters}

Using Eq. 3, we calculate the spin parameters for $l=1, m=1$ g modes, $l=2, m=2 \mathrm{~g}$ modes, and $k=-2, m=-1 \mathrm{r}$ modes. Figure 19 displays their distributions. For $l=1, m=1 \mathrm{~g}$ modes, the spin parameters show a rapid rise and a slow drop from 0 to 15 . Most of the modes have $s$ around 5 . Several slow rotators have extremely low spin parameters, which form the peak close to zero. For $l=2, m=2 \mathrm{~g}$ modes, the spin parameters are lower since the pulsation frequencies are longer than dipole g modes. Most of them are around 2.5. For $\mathrm{r}$ modes with $k=-2, m=-1$, they show different spin parameter distributions. The smallest spin parameter value is $\sim 6$ and the highest is $\sim 35$. They show a peak around 9 . The r-mode spin parameters are typically larger than that of $g$ modes and have different distributions, implying diverse pulsation properties for them. 


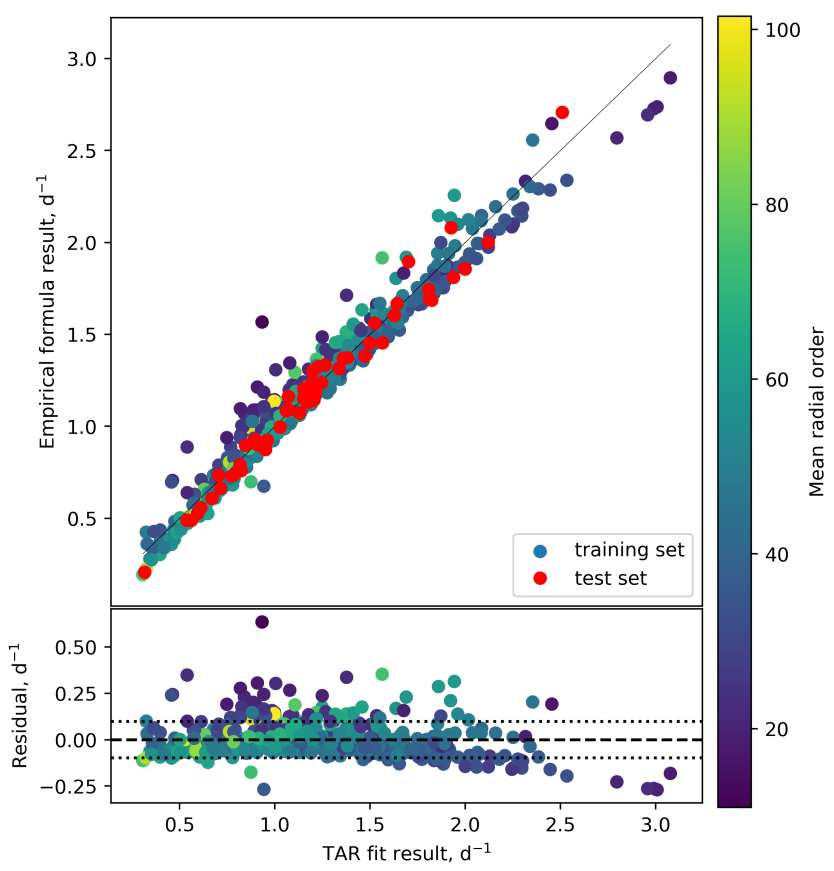

Figure 20. The near-core rotation rates calculated by eq. 5 for $k=0, m=1 \mathrm{~g}$ modes. Upper panel: the relation between the input and predicted near-core rotation rates. The red circles are the test set while the others are the training set. Lower panel: the fit residuals. The residuals have the standard deviation of $0.1 \mathrm{~d}^{-1}$, which is the accuracy of eq. 5 .

\section{ROTATION ON S-P DIAGRAM}

\subsection{Empirical method to calculate rotation rate}

The fit of the TAR reveals the near-core rotation rate, asymptotic spacing, and the estimated radial orders of one pattern, given the quantum numbers $k$ and $m$. This fitting procedure converges faster with a good initial estimate of $f_{\text {rot. }}$ Hence, we report an empirical method to estimate the near-core rotation rate based on the three observables: the mean period $\langle P\rangle$, the mean spacing $\langle\Delta P\rangle$, and the slope $\Sigma$. We use a simple formula to describe the relation between the near-core rotation rate and these three observables. The formula is designed as:

$f_{\mathrm{rot}}=\frac{A}{\langle P\rangle}+\frac{B}{\langle\Delta P\rangle}+C \Sigma+D$

where $A, B, C$, and $D$ are the coefficients. We selected inversely proportional functions for $\langle P\rangle$ and $\langle\Delta P\rangle$ (both in unit of days) because they are inversely correlated with the rotation rate. For the slope $\Sigma$, the proportional relation is used since rapid rotation causes a steeper period-spacing pattern. On the left hand side of eq. 5 , the unit of $f_{\text {rot }}$ is $\mathrm{d}^{-1}$. On the right hand side, the coefficients $A$ and $B$ are dimensionless, the unit of coefficient $C$ and $D$ are $\mathrm{d}^{-1}$. We applied eq. 5 to $l=1, m=1 \mathrm{~g}$ modes, $l=2, m=2$ g modes, and $k=-2, m=-1 \mathrm{r}$ modes, respectively. The slow rotators with $f_{\text {rot }}<0.4 \mathrm{~d}^{-1}$ were excluded, since the slope is affected by the glitches more than the rotational effect. The best-fitting coefficients are listed in Table 2.

Figure 20 shows the fit result of $k=0, m=1 \mathrm{~g}$ modes.
Table 2. The coefficients of eq. 5 for $k=0, m=1 \mathrm{~g}$ mode, $k=$ $0, m=2 \mathrm{~g}$ mode, and $k=-2, m=-1 \mathrm{r}$ mode. Note that $k=l-|m|$ for g modes. $A, B, C$, and $D$ are the coefficients. $\delta f_{\text {rot }}$ is the fitting accuracy in unit of $\mathrm{d}^{-1}$.

\begin{tabular}{rrrrrr}
\hline$(k, m)$ & $A$ & $B$ & $C, \mathrm{~d}^{-1}$ & $D, \mathrm{~d}^{-1}$ & $\delta f_{\text {rot }}, \mathrm{d}^{-1}$ \\
\hline$(0,1)$ & 0.4189 & 0.001603 & -11.75 & -0.3554 & 0.1 \\
$(0,2)$ & 0.3346 & 0.0003965 & -2.477 & -0.2462 & 0.07 \\
$(-2,-1)$ & 1.167 & -0.0002585 & -0.2360 & 0.1099 & 0.03 \\
\hline
\end{tabular}

Table 3. The parameters of the simulated curvatures in Fig. 21. $k$ and $m$ are the quantum numbers of the modes. All the patterns have 20 modes with different radial order centres $\langle n\rangle . \Pi_{0}$ is the asymptotic spacing and $f_{\text {rot }}$ is the near-core rotation rate.

\begin{tabular}{rrrrr}
\hline Colour & $(k, m)$ & $\langle n\rangle$ & $\begin{array}{r}\Pi_{0}, \mathrm{~s} \\
\min , \max , \text { step }\end{array}$ & $\begin{array}{r}f_{\text {rot }}, \mathrm{d}^{-1} \\
\min , \max , \text { step }\end{array}$ \\
\hline Blue & $(0,1)$ & 20 & $3600,5600,200$ & $0.0,4.0,0.1$ \\
Cyan & $(0,1)$ & 40 & $3600,5600,200$ & $0.0,4.0,0.1$ \\
Green & $(0,2)$ & 20 & $3600,5600,200$ & $0.0,4.0,0.1$ \\
Red & $(-2,-1)$ & 20 & $3600,5600,200$ & $0.7,4.0,0.1$ \\
\hline
\end{tabular}

The upper panel reveals the correlation of near-core rotation rates from the TAR fit and the empirical formula eq. 5 . We selected $90 \%$ of the data points (coloured by their mean radial orders) as the training set to obtain the coefficients (listed in the first line of Table 2), and use the other 10\% (red circles) to test if the coefficients work well and to avoid overfitting. Both the training set and the test set show a positive correlation, which means that eq. 5 with the parameters in Table 2 can estimate the near-core rotation rate. The lower panel shows the differences between the input and output rotation rates. The differences have a standard deviation of $0.1 \mathrm{~d}^{-1}$, which is the precision of eq. 5 for $k=0, m=1 \mathrm{~g}$ modes. We find that the mean radial orders show a gradient, in the sense that the points with small residuals have larger mean radial orders than those with large differences. Hence the scatter of eq. 5 is partially caused by the mean radial order.

The second and third lines in Table 2 list the coefficients of eq. 5 but for $k=0, m=2 \mathrm{~g}$ modes and $k=-2, m=-1$ $\mathrm{r}$ modes. The precision of eq. 5 for $k=0, m=2 \mathrm{~g}$ modes is $0.07 \mathrm{~d}^{-1}$, which is similar to the $k=0, m=1 \mathrm{~g}$-mode residuals. However, The precision of $k=-2, m=-1 \mathrm{r}$ modes is $0.03 \mathrm{~d}^{-1}$, significantly smaller that those of $g$ modes. The reason is that $r$ modes are not affected by the range of radial orders as much as g modes.

Equation 5 with the coefficients in Table 2 gives the relation between the rotation rate and the three observables. They can be used as the estimate of the near-core rotation rate before running the TAR fit code. We also tried to search the formula for asymptotic spacing $\Pi_{0}$ and mean radial order as a function of $\langle P\rangle,\langle\Delta P\rangle$ and $\Sigma$, but there are no clear correlations.

\subsection{Rotation on the $\mathrm{S}-\mathrm{P}$ diagram}

To plot the rotation rate on the $\mathrm{S}-\mathrm{P}$ diagram, we used the TAR to simulate the period-spacing pattern and calculate the synthetic mean periods and slopes. We show our simu- 


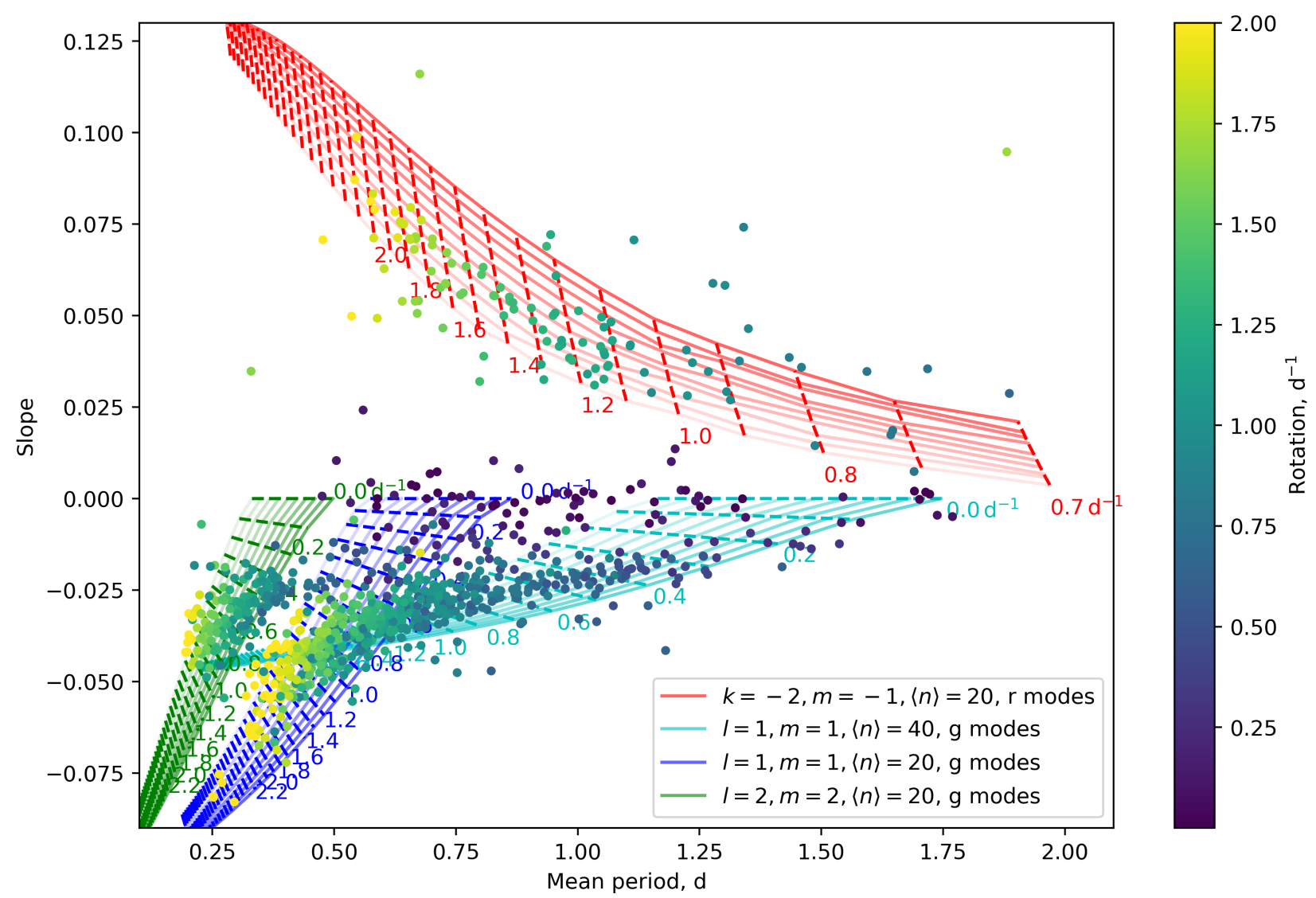

Figure 21. Theoretical S-P diagram. One solid line has the same quantum numbers, asymptotic spacing, and radial order centres with rotation increasing from right to left. The dashed lines connect the positions with same rotation rate. Different colours show the curves with different quantum numbers. The transparency stands for the asymptotic spacing $\Pi_{0}$. The lighter, the smaller the $\Pi_{0}$. The numbers show the rotation rates in $\mathrm{d}^{-1}$ of the nearest dashed lines. The parameters for those curves are listed in Table 3. The data points are coloured by their near-core rotation rates to make the comparison with the simulated rotation straightforward.

lated curves in Fig. 21, whose parameters are listed in Table 3 . For both $g$ and $r$ modes, the number of modes in each pattern was selected as 20 and the range of $\Pi_{0}$ is from $3600 \mathrm{~s}$ to $5600 \mathrm{~s}$ with step of $200 \mathrm{~s}$. For g modes, the rotation is between 0 to $4.0 \mathrm{~d}^{-1}$ with step of $0.1 \mathrm{~d}^{-1}$ to cover the observed range. Two regions of radial orders were used for g modes, centred at $20(10<n<30)$ and $40(30<n<50)$, as shown in blue and cyan lines in Fig. 21. For $\mathrm{r}$ modes, only radial orders around $20(10<n<30)$ and $0.7 \mathrm{~d}^{-1}<f_{\text {rot }}<4.0 \mathrm{~d}^{-1}$ are displayed because these parameters regions can explain the $\mathrm{r}$ modes data well.

Figure 21 shows the simulated results of the S-P diagram. We find that the simulated curves cover the data points well. For $l=1, m=1 \mathrm{~g}$ modes, the data show two trends: one is the patterns with lower radial orders (the blue curves) which show shorter mean periods and the steeper relations between the slope and the period; another trend shows higher radial orders (cyan curves) whose mean periods are generally longer and the relation between the slope and the period is flatter. Two trends have an overlap over $P \sim 0.5 \mathrm{~d}$. For $l=2, m=2 \mathrm{~g}$ modes, the trend between the slope and the mean period is not obvious due to the limited detection of quadrupole g modes. So only $10<n<30$ is used to cover $l=2, m=2 \mathrm{~g}$ modes.

The S-P diagram is a map for the near-core rotation rate, as marked by the dashed lines and numbers in Fig. 21. The dashed lines connect the positions with same rotation rates, hence we can estimate the near-core rotation rate by placing the star on the $\mathrm{S}-\mathrm{P}$ diagram. The rapidly rotating stars generally appear on the left in the S-P diagram, because of both the Coriolis force and the transformation between the co-rotating and inertial reference frame. The estimate of the near-core rotation rate is affected by the mode identification, the asymptotic spacing, and the radial orders. There is an overlapping area around $P \sim 0.5 \mathrm{~d}$. In this area, the pattern with higher radial order (cyan curves) shows a higher rotation rate $\left(\sim 1.6 \mathrm{~d}^{-1}\right)$ while the pattern with low radial order (blue curves) has a slower rotation rate $\left(\sim 1 \mathrm{~d}^{-1}\right)$. For $\mathrm{r}$ modes, the relation is clearer and the S-P map can give a better estimate for the near-core rotation rate. It also explains why the r-mode residuals in Table 2 are smaller than those for the g-mode, since there is only one trend for $\mathrm{r}$ modes on the S-P diagram. 
Table 4. The slopes and splittings of 11 rapidly-rotating stars. $\delta v$ are the mean splittings. $\Sigma_{l, m}$ are the slopes. The uncertainty on the last digit is given between brackets.

\begin{tabular}{rrrr}
\hline $\mathrm{KIC}$ & $\delta \boldsymbol{v}, \mathrm{d}^{-1}$ & $\Sigma_{l=1, m=1}$ & $\Sigma_{l=1, m=0}$ \\
\hline 3348714 & $0.38(2)$ & $-0.0352(2)$ & $-0.0154(4)$ \\
4285040 & $0.46(2)$ & $-0.014(4)$ & $-0.0163(3)$ \\
4846809 & $0.65(1)$ & $-0.036(6)$ & $-0.0087(7)$ \\
4952246 & $0.316(9)$ & $-0.005(5)$ & $-0.011(1)$ \\
5476473 & $1.00(2)$ & $-0.055(5)$ & $-0.0148(8)$ \\
7701947 & $0.32(1)$ & $-0.0281(1)$ & $-0.0165(5)$ \\
7778114 & $0.50(2)$ & $-0.0355(5)$ & $-0.0202(2)$ \\
8523871 & $0.32(5)$ & $-0.075(5)$ & $-0.067(7)$ \\
9595743 & $0.42(1)$ & $-0.044(4)$ & $-0.027(7)$ \\
12102187 & $0.291(6)$ & $-0.017(7)$ & $-0.019(9)$ \\
12401800 & $0.43(1)$ & $-0.0308(8)$ & $-0.021(1)$ \\
\hline
\end{tabular}

\section{FAST ROTATORS WITH SPLITTINGS}

Li et al. (2019a) reported $22 \gamma$ Dor stars in which rotational splittings were seen. The rotation rates of those stars are generally slow (with splitting smaller than $0.2 \mathrm{~d}^{-1}$ ), hence their period-spacing patterns with different azimuthal orders $m$ overlap each other. The traditional échelle diagram was used to distinguish the patterns and the shift-copy method helped match the modes with equal radial order $n$ (see details in Li et al. 2019a).

In this work, we found 11 stars whose splittings are much larger. Figure 22 displays the splittings of KIC 7701947 as an example. The top panel shows the power spectrum, in which the red dots are the extracted frequencies and the open dots are the likely combination frequencies. We mark the $l=1, m=1 \mathrm{~g}$ modes as the blue vertical lines and the $l=1, m=0 \mathrm{~g}$ modes as the green vertical lines. The plus and circle symbols mark the locations of $m=1$ and $m=0$ modes, respectively. The horizontal dashed lines connect the modes with equal $n$. The bottom panel shows two period-spacing patterns, the left one (blue plus) is $l=1, m=1 \mathrm{~g}$ modes and the right one (green circle) is $l=1, m=0 \mathrm{~g}$ modes. The period-spacing patterns look similar to those in Fig. 1 but two features expose the difference:

- the ratio of pulsation periods between $m=1$ and $m=0$ modes does not have a factor of two.

- Slopes of $m=1$ and $m=0$ modes are different so the patterns are not parallel.

We also state that these splitting stars are not binaries, since we can use the same parameters $\left(\Pi_{0}, f_{\text {rot }}\right)$ to fit both the patterns $(m=1$ or $m=0)$ in each star.

Under the condition of slow rotation, the dipole $(l=$ 1) g-mode splitting is calculated based on the first-order perturbation

$\delta f=f_{n, l=1, m=1}-f_{n, l=1, m=0}=m\left(1-C_{n, l}\right) f_{\text {rot }}$,

where $f$ is the pulsation frequency, $C_{n, l} \simeq 1 /[l(l+1)]=0.5$ is the Ledoux constant, $f_{\text {rot }}$ is the near-core rotation rate (Ledoux 1951), the term $m$ is one since we consider dipole g modes. We only consider the splitting between $m=1$ and $m=0$ modes since $m=-1$ retrograde modes are absent in the fast rotating stars (see theory in e.g. Saio et al. 2018b). However, the perturbation is broken with increasing rotation rate. For the newly-discovered splitting stars with much faster rotation rates, the splittings vary between different overtones significantly and it is hard to match the modes with the same $n$.

For the fast rotators, the TAR in Eq. 2 is a good approximation. The frequency in the co-rotating frame is

$f_{n l m, \mathrm{co}}=\frac{\sqrt{\lambda_{l, m, s}}}{\Pi_{0}\left(n+\varepsilon_{g}\right)}$,

whose variables are same as Eq. 2. The frequency in the inertial frame is

$f_{\text {nlm, in }}=f_{\text {nlm,co }}+m f_{\text {rot }}$,

where $f_{\text {rot }}$ is the near-core rotation rate. Therefore the splittings is calculated by

$\delta f=m f_{\mathrm{rot}}+\frac{1}{\Pi_{0}\left(n+\varepsilon_{g}\right)}\left(\sqrt{\lambda_{l, m=1, s}}-\sqrt{\lambda_{l, m=0, s^{\prime}}}\right)$.

Hence the Ledoux 'constant' $C=1-\delta f /\left(m f_{\text {rot }}\right)$ is no longer a 'constant' (see also Keen et al. 2015; Murphy et al. 2016). Figure 23 shows the theoretical result of the varying Ledoux 'constant'. The parameters are $\Pi_{0}=4000 \mathrm{~s}$ and $f_{\text {rot }}=1 \mathrm{~d}^{-1}$, which are chosen from the distributions shown in Fig. 9 and Fig. 13. We find that with increasing pulsation frequency, the Ledoux 'constant' increases from $\sim 0.485$, reaches the highest value around 0.52 , and drops slowly. The deviation from the first-order perturbation is $\sim \pm 0.02$. We also evaluated different values for $\varepsilon_{g}$ and find that it does not change the curve, but only shifts the frequencies of zonal modes, as shown by the blue circles and yellow squares in Fig. 23.

Figure 24 shows the comparison between the observed splittings and the theoretical predictions of KIC 7701947. The blue curve and shaded area show the theoretical splittings and uncertainties, whose parameters are from the bestfitting TAR result. We tried to introduce an artificial shift on the radial order of the $l=1, m=0 \mathrm{~g}$ modes and plot the results as grey circles and triangles in Fig. 24. The black squares are the matching that follows the theory best. We find that changing the mode matching by $\Delta n= \pm 1$ indeed changes the shape of splitting. The splittings with correct matching generally follow the theoretical prediction. However, the observed splittings are higher than the theoretical one, showing a discrepancy with the theory. It means the best model which fits the period-spacing patterns can only partly explain the splittings. This reflects the limit of the asymptotic formula of the TAR in Eq. 2, which can be improved by performing a full seismic calculation.

Ouazzani et al. (2017) showed that the near-core rotation can be deduced roughly by the slope. To make an observed relation between rotation and slope, we combined the 11 rapidly rotating stars with splittings and 22 slow rotating stars from Li et al. (2019a) to extend the observed slope-splitting diagram to a larger splitting area. Figure 25 shows the slope-splitting relation with both the observations and theoretical curves. The observed points cluster into two groups: the left one is composed of the slow rotators with splitting smaller than $0.2 \mathrm{~d}^{-1}$ and slope close to 0 , while the points with splitting larger than $0.2 \mathrm{~d}^{-1}$ are the fast rotators. There is a gap over $0.2 \mathrm{~d}^{-1}$, which corresponds to the boundary between the slow and fast rotators in Section 4.2. Due to the effect of rotation, the slopes of the fast rotators deviate from zero and become lower than the slow rotators. The slopes of zonal modes (green) are generally flatter than 

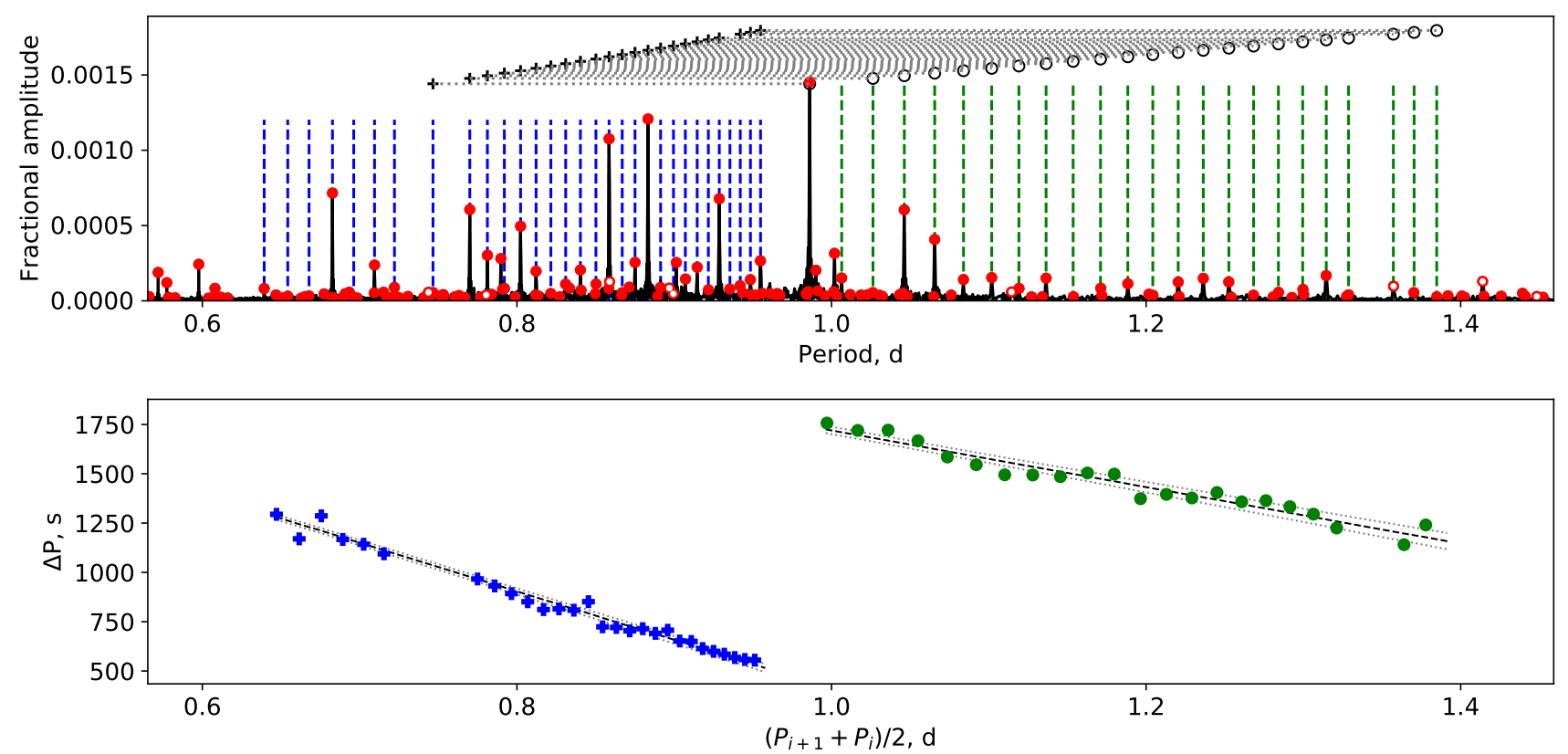

Figure 22. The amplitude spectrum and period-spacing patterns of KIC 7701947. Top panel: the amplitude spectrum as a function of period. The red dots are the extracted frequencies and the open dots show the likely combination frequencies. The vertical dashed lines show the linear fit by Eq. 1. Two period-spacing patterns are seen. The left one denoted by the blue dashed lines is the $l=1, m=1 \mathrm{~g}$ modes. The right one (green vertical lines) is the $l=1, m=0 \mathrm{~g}$ modes. Above the spectrum ' + ' is the $m=1$ modes and ' $\mathrm{O}$ ' is the $m=0$ modes. The horizontal dotted lines connect the modes with same radial order $n$. Bottom: the period spacing patterns. The left one is the $l=1, m=1 \mathrm{~g}$ modes while the right one is $l=1, m=0 \mathrm{~g}$ modes. The linear fits and uncertainties are shown by the black and grey dashed lines, with dips removed.

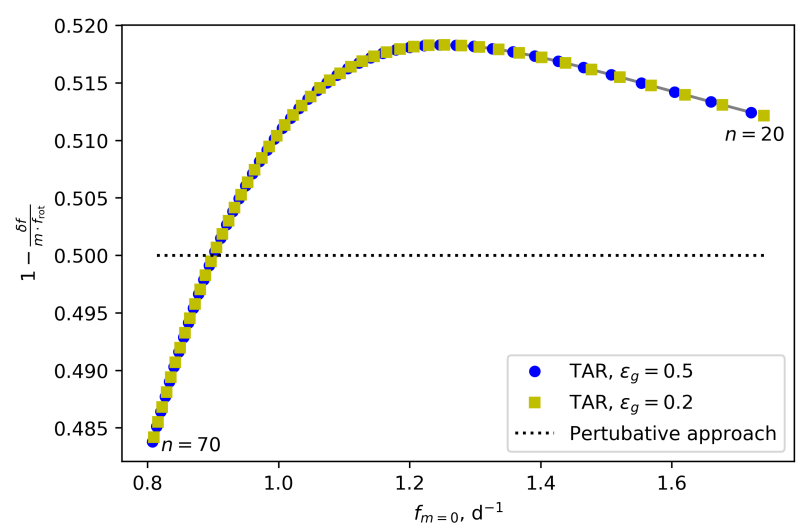

Figure 23. The theoretical Ledoux 'constant' as a function of frequency from the TAR, with $\Pi_{0}=4000 \mathrm{~s}$ and $f_{\text {rot }}=1 \mathrm{~d}^{-1}$. The considered radial orders have values between 20 and 70 . The blue circles and yellow squares show the 'constant' with different $\varepsilon_{g}$. The horizontal dotted line denotes the position with $C_{n, l}=0.5$.

the slopes of dipole sectoral modes (blue), consistent with the theoretical curves by Ouazzani et al. (2017) and us. The retrograde modes (red) are only seen in the slow rotators. The reasons of the absence of retrograde modes in fast rotators are: (1) the period spacings are around $10^{4} \mathrm{~s}$ hence they are hard to detect; (2) the amplitudes of retrograde modes are concentrated to the equator; (3) an additional latitudi-

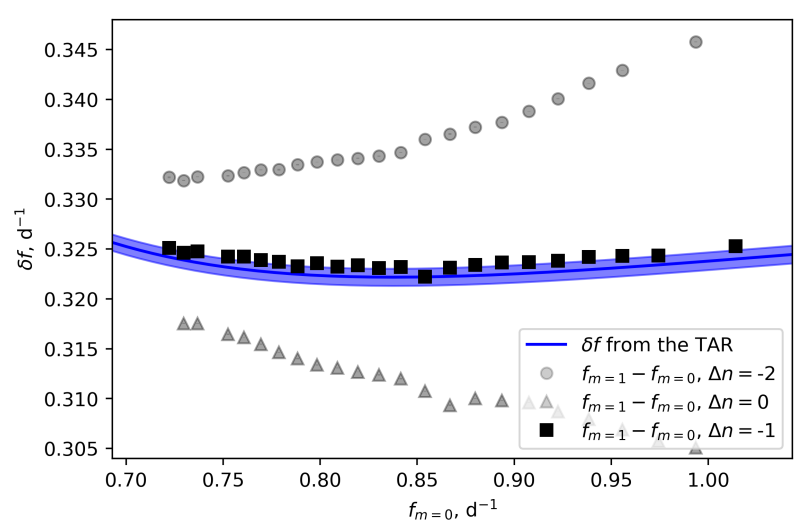

Figure 24. The splitting variation as a function of frequency of KIC 7701947. The blue line and shaded area are the predicted curve and uncertainty from the TAR fitting result. The black squares are the splittings which follow the theory best, whose radial orders are subtracted by 1 artificially for the best-fitting result. The grey circles and triangles are the splittings whose radial orders are mismatched by a factor of \pm 1 around -1 .

nal nodal lines appear when $s>1$ hence retrograde modes become tesseral modes. Therefore, no retrograde modes are expected in fast rotators (Saio et al. 2018b).

The shaded areas are the theoretical regions of slopes as a function of splittings with different modes. We calculated 


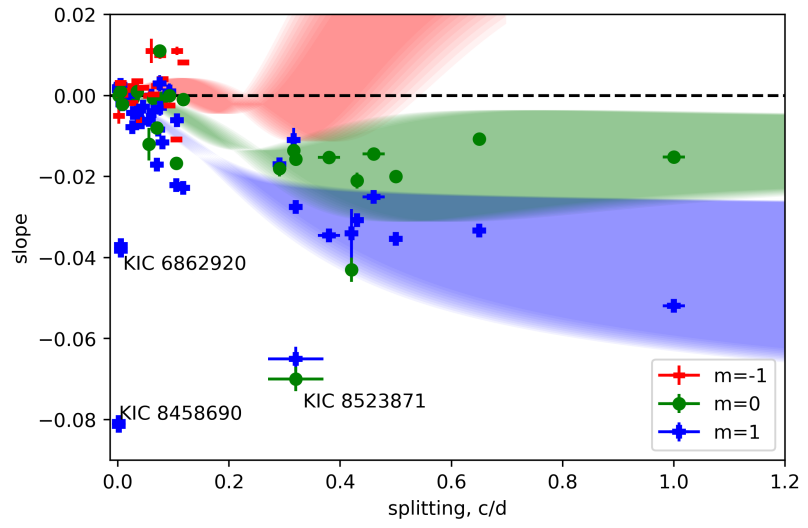

Figure 25. Observed relation between slope and splitting from 34 stars. The shaded areas are theoretical curves. We consider the radial orders from 20 to 70 to make the theoretical areas. KIC 6862920 and KIC 8458690 are binaries, KIC 8523871 shows a much larger asymptotic spacing, hence they become outliers.

the simulated period-spacing patterns by the TAR, and measured the slopes of them. The asymptotic spacing $\Pi_{0}$ is from $3500 \mathrm{~s}$ to $4500 \mathrm{~s}$ with step of $100 \mathrm{~s}$. The radial order is from 20 to 70 . Since the period spacing changes quasi-linearly with period, the slope changes as a function of radial order. We measured the slope at the beginning and at the end of each simulated period-spacing pattern. We found that the slope variation at different radial orders is significant, hence the slope-splitting curves in our work show a much larger dispersion than Ouazzani et al. (2017), who neglected this effect. Our simulated curves in Fig. 25 cover the data points in the fast rotation area, but have difficulty in the slow rotation area. The slopes of slowly-rotating stars spread much wider than the shaded area, implying that dips dominate the measurement of slopes in slowly-rotating stars.

We only see 11 rapidly rotating stars with splittings among $611 \gamma$ Dor stars in our sample. The lack may reflect the surface amplitude distribution of tesseral modes. With increasing rotation, the mode geometry are concentrated toward the equator, hence the brightness change by pulsations is cancelled out unless the line of sight is almost aligned with the rotation axis of the star (Townsend 2003b). Apart from these 11 splitting stars, we also find two stars, KIC 5092684 and KIC 5544996, which show $l=2, m=1$ modes. Precise observations of their projected equatorial velocities $v \sin i$ will reveal their inclinations and allow us to evaluate the theoretical expectations for the amplitude distributions of the pulsation modes.

\section{SURFACE MODULATIONS}

The $\mathrm{g}$ and $\mathrm{r}$ modes allow us to measure the rotation rate of the near-core region. To see how the rotation rate changes radially, which is crucial to understand the angular momentum transfer, we looked for the surface modulation signals. The surface modulation might be caused by spots (e.g. McQuillan et al. 2014), or stellar activities, which is straightforward to detect since the signal is located within the typical $\mathrm{g}$ mode period region. Although we excluded the eclipsing
Table 5. The near-core and surface rotation rates, and their ratios of 58 stars which show surface modulation signals. 'EB' means the star is an eclipsing binary and 'SURF' means the signal is caused by surface modulations. $f_{\text {core }}$ is the near-core rotation rate and $f_{\text {surf }}$ is the surface rotation rate.

\begin{tabular}{|c|c|c|c|c|}
\hline $\mathrm{KIC}$ & Type & $f_{\text {core }}, \mathrm{d}^{-1}$ & $f_{\text {surf }}, \mathrm{d}^{-1}$ & $f_{\text {core }} / f_{\text {surf }}$ \\
\hline KIC 2846358 & SURF & $0.755(4)$ & $0.754(8)$ & $1.00(1)$ \\
\hline KIC 3341457 & EB & $1.859(1)$ & $1.893(7)$ & $0.982(4)$ \\
\hline KIC 3440840 & SURF & $0.93(6)$ & $0.938(7)$ & $0.99(6)$ \\
\hline KIC 3967085 & SURF & $0.76(2)$ & $0.77(1)$ & $1.00(3)$ \\
\hline KIC 4171102 & SURF & $0.7579(6)$ & $0.76(2)$ & $1.00(2)$ \\
\hline KIC 4567531 & SURF & $1.018(5)$ & $0.94(2)$ & $1.08(2)$ \\
\hline KIC 4932417 & SURF & $1.285(8)$ & $1.23(2)$ & $1.05(2)$ \\
\hline KIC 4951030 & SURF & $2.53(3)$ & $2.49(4)$ & $1.02(2)$ \\
\hline KIC 5021329 & SURF & $2.02(2)$ & $1.98(4)$ & $1.02(2)$ \\
\hline KIC 5025464 & SURF & $1.57(3)$ & $1.561(3)$ & $1.00(2)$ \\
\hline KIC 5115637 & SURF & $0.714(6)$ & $0.72(2)$ & $0.99(2)$ \\
\hline KIC 5210153 & SURF & $1.051(4)$ & $1.040(5)$ & $1.011(6)$ \\
\hline KIC 5370431 & SURF & $0.618(5)$ & $0.6122(6)$ & $1.009(8)$ \\
\hline KIC 5374279 & SURF & $1.00(2)$ & $0.873(4)$ & $1.14(3)$ \\
\hline KIC 5608334 & SURF & $2.25(1)$ & $2.241(3)$ & $1.005(6)$ \\
\hline KIC 5652678 & SURF & $1.140(5)$ & $1.163(6)$ & $0.980(6)$ \\
\hline KIC 5876187 & SURF & $0.596(5)$ & $0.584(4)$ & $1.02(1)$ \\
\hline KIC 5954264 & SURF & $1.330(9)$ & $1.329(6)$ & $1.001(8)$ \\
\hline KIC 5978913 & SURF & $0.955(5)$ & $0.950(6)$ & $1.006(8)$ \\
\hline KIC 6041803 & EB & $1.53(2)$ & $1.524(7)$ & $1.00(1)$ \\
\hline KIC 6284209 & SURF & $1.48(2)$ & $1.42(4)$ & $1.04(3)$ \\
\hline KIC 6366512 & SURF & $1.17(1)$ & $1.18(1)$ & $0.99(1)$ \\
\hline KIC 6445969 & SURF & $1.263(7)$ & $1.244(5)$ & $1.015(7)$ \\
\hline KIC 6469690 & SURF & $0.566(5)$ & $0.554(6)$ & $1.02(1)$ \\
\hline KIC 6935014 & SURF & $0.789(3)$ & $0.788(7)$ & $1.00(1)$ \\
\hline KIC 7059699 & SURF & $2.30(2)$ & $2.27(2)$ & $1.01(1)$ \\
\hline KIC 7287165 & SURF & $0.95(1)$ & $0.986(2)$ & $0.96(1)$ \\
\hline KIC 7344999 & SURF & $1.40(1)$ & $1.40(3)$ & $1.00(2)$ \\
\hline KIC 7434470 & EB & $1.77(1)$ & $1.698(1)$ & $1.044(6)$ \\
\hline KIC 7596250 & EB & $1.1876(7)$ & $1.185(4)$ & $1.003(4)$ \\
\hline KIC 7620654 & SURF & $1.88(2)$ & $1.83(4)$ & $1.03(2)$ \\
\hline KIC 7621649 & SURF & $0.7745(4)$ & $0.7802(6)$ & $0.9928(9)$ \\
\hline KIC 7840642 & SURF & $1.158(6)$ & $1.158(6)$ & $1.000(8)$ \\
\hline KIC 7968803 & SURF & 1.94(1) & $1.949(7)$ & $0.997(8)$ \\
\hline KIC 8180062 & SURF & $0.907(5)$ & $0.904(9)$ & $1.00(1)$ \\
\hline KIC 8197019 & SURF & $1.89(5)$ & $1.86(4)$ & $1.01(3)$ \\
\hline KIC 8264667 & SURF & $0.684(5)$ & $0.682(2)$ & $1.002(8)$ \\
\hline KIC 8264708 & SURF & $1.636(1)$ & $1.636(1)$ & $1.000(1)$ \\
\hline KIC 8293692 & SURF & $1.00(2)$ & $1.044(9)$ & $0.95(2)$ \\
\hline KIC 9573582 & SURF & $0.9495(5)$ & $0.946(5)$ & $1.004(6)$ \\
\hline KIC 9652302 & SURF & $0.9147(6)$ & $0.910(2)$ & $1.005(3)$ \\
\hline KIC 9716350 & SURF & $0.863(3)$ & $0.864(1)$ & $0.999(3)$ \\
\hline KIC 9716563 & SURF & $0.9081(9)$ & $0.90(2)$ & $1.01(2)$ \\
\hline KIC 9847243 & SURF & $0.933(5)$ & $0.913(9)$ & $1.02(1)$ \\
\hline KIC 10347481 & SURF & $1.303(5)$ & $1.27(2)$ & $1.03(2)$ \\
\hline KIC 10423501 & SURF & $0.8420(6)$ & $0.841(4)$ & $1.001(4)$ \\
\hline KIC 10470294 & SURF & $2.00(2)$ & $1.96(4)$ & $1.02(2)$ \\
\hline KIC 10483230 & SURF & $0.9126(4)$ & $0.918(2)$ & $0.995(2)$ \\
\hline KIC 10669515 & SURF & $1.596(7)$ & $1.567(4)$ & $1.019(5)$ \\
\hline KIC 10803371 & EB & $0.98(2)$ & $1.011(3)$ & $0.97(2)$ \\
\hline KIC 11183399 & SURF & $1.89(2)$ & $1.868(3)$ & $1.01(1)$ \\
\hline KIC 11201483 & SURF & $2.28(2)$ & $2.23(3)$ & $1.02(2)$ \\
\hline KIC 11294808 & SURF & $0.802(5)$ & $0.7887(7)$ & $1.016(7)$ \\
\hline KIC 11395936 & EB & $0.944(7)$ & $0.953(6)$ & $0.99(1)$ \\
\hline KIC 11462151 & SURF & $2.00(2)$ & $1.93(4)$ & $1.03(2)$ \\
\hline KIC 11520274 & SURF & $1.03(2)$ & $1.025(3)$ & $1.00(2)$ \\
\hline KIC 11922283 & SURF & $1.088(8)$ & $1.07(1)$ & $1.02(1)$ \\
\hline KIC 12202137 & SURF & $1.96(1)$ & $1.96(1)$ & $0.998(8)$ \\
\hline
\end{tabular}




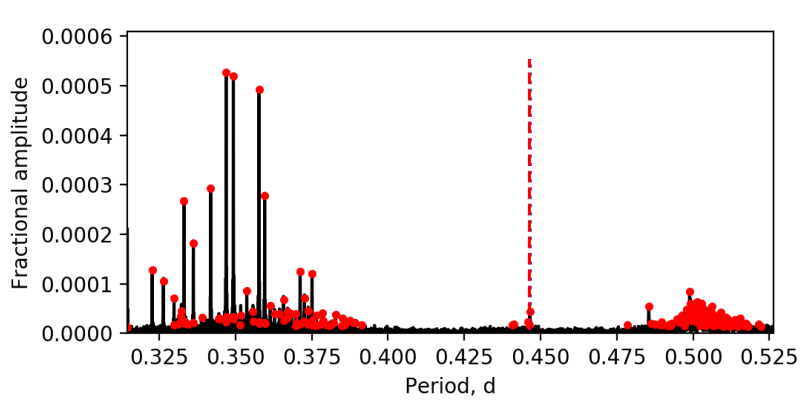

Figure 26. The near-core and surface rotations of KIC 5608334 . The red dots show the frequencies. The red dashed line shows the surface rotation period, which is consistent with the near-core rotation rate derived by the g-mode pattern. The hump at $0.5 \mathrm{~d}$ is the unsolved $r$ modes.

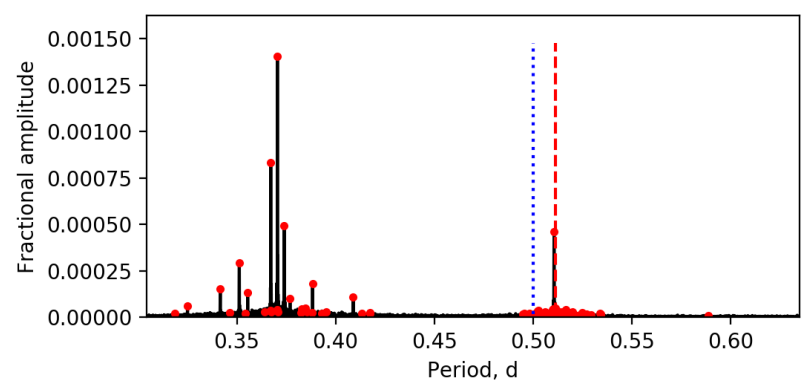

Figure 27. Same as Fig. 26 but for KIC 10470294 . The red vertical dashed line shows the surface rotation period while the blue dotted line gives the near-core rotation rate. We identify that in this star the hump at $0.52 \mathrm{~d}$ is the surface rotation signal, not $\mathrm{r}$ modes.

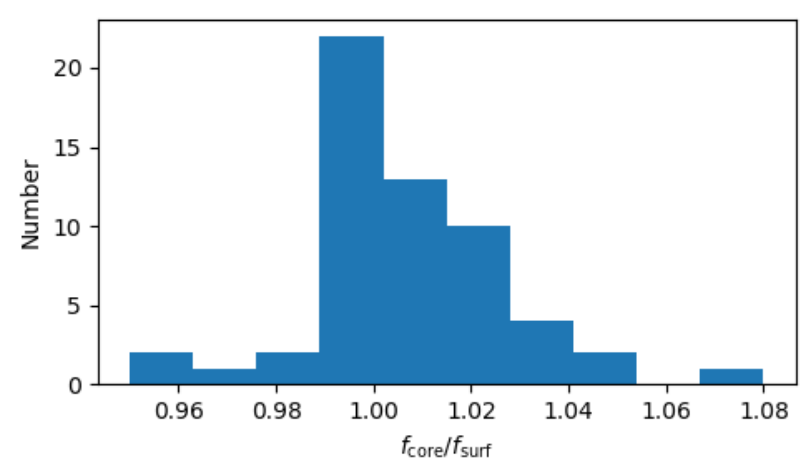

Figure 28. The distributions of the core-to-surface rotation ratio.

binaries (EB) when selecting the stars, there are still several stars which were classified as EB by Kirk et al. (2016), since their eclipses are too shallow to be seen in the time domain. The orbital periods are equal to the surface rotation periods for short period EBs, considering the components are tidally locked. Hence we can use the orbital period as the surface rotation period. We follow the criteria used by Van Reeth et al. (2018) and Li et al. (2019b) to select the rotational modulation signal. The criteria are:

- the surface modulation is a closely-spaced group of peaks in the amplitude spectrum, assuming the lifetime of spot is shorter than the 4-yr observation.

- the signal-to-noise ratio $(\mathrm{S} / \mathrm{N})$ is larger than 4 .

- the harmonic of the highest peak is seen.

- the signal is located between $g$ and $r$ modes. For those without $r$ modes, we search the signal between one and two times the g-modes mean period, because the mean period of $r$ modes is approximately twice the mean period of g modes.

- Both the rotational modulation signal at the rotation frequency and its harmonic have to be well-separated from the period-spacing patterns, to avoid mistaking pulsation modes for rotational modulation.

We find 58 stars which show surface modulations, representing about $9.5 \%$ of the 611 stars. The fraction is consistent with spectroscopic observations of Zeeman splitting (Donati \& Landstreet 2009; Wade et al. 2016) and previous photometric observations of smaller samples (Van Reeth et al. 2018; Li et al. 2019b). Table 5 lists the KIC numbers of these stars, the near-core $\left(f_{\text {core }}\right)$ and surface $\left(f_{\text {surf }}\right)$ rotation rates, and their ratios. Figure 26 gives the amplitude spectrum of KIC 5608334 which shows a surface rotation signal. We find a peak group at $\sim 0.44 \mathrm{~d}$ (red dashed line), which is consistent with the near-core rotation rate derived by the g-mode period-spacing pattern. A hump in Fig. 26 appears around $0.5 \mathrm{~d}$, which we identify as unresolved $\mathrm{r}$ modes (e.g. Saio et al. 2018a).

However, not all such humps are $\mathrm{r}$ modes. Figure 27 displays the surface modulation of KIC 5608334, in which the hump at $\sim 0.52 \mathrm{~d}$ lies over the near-core rotation period. This hump is not unresolved $\mathrm{r}$ modes since the latter have periods longer than the rotation period. We conclude that this hump is caused by the spots on the surface, as pointed out by e.g. Balona (2013).

We calculate the ratio between the near-core and surface rotation and show them in Fig. 28. We find that almost all the stars rotate nearly rigidly since the ratio is between 0.95 and 1.08. The distribution shows a sharp drop at $\sim 0.99$, implying that the near-core region rotates slightly faster than the surface, which is consistent with the theoretical rotation profile reported by Rieutord (2006). A possible selection bias needs to be mentioned. A surface rotation period longer than the near-core rotation period might not be detected since this signal may be identified as a peak group of unresolved $r$ modes.

\section{CONCLUSIONS}

We report 960 period-spacing patterns detected from 611 $\gamma$ Dor stars, including 22 slow rotators with rotational splittings, 11 rapid rotators with rotational splittings, 110 stars with $\mathrm{r}$ modes, and 58 stars that present surface modulation signals. The majority $(62.0 \%)$ of the detected modes are $l=1, m=1 \mathrm{~g}$ modes. We also see many $l=2, m=2$ g modes and $k=-2, m=-1$ Rossby modes, which comprise $18.9 \%$ and $11.5 \%$ of the sample, respectively. Among the $611 \gamma$ Dor stars, there are 339 stars which only show dipole $\mathrm{g}$ modes, 145 stars showing both dipole and quadrupole $\mathrm{g}$ 
modes, 83 stars showing dipole $\mathrm{g}$ modes and $k=-2, m=-1$ $r$ modes, 27 stars showing dipole, quadrupole g modes, and $r$ modes. We also find 16 stars whose dipole g modes cannot be resolved, and one star which does not show any dipole $g$ mode power.

For each pattern, a series of pulsation periods were identified. The mean periods, the mean period spacings, and the slope were calculated for each pattern. We find $\gamma$ Dor stars have a relation on the slope-mean period diagram $(\mathrm{S}-\mathrm{P}$ diagram, Fig. 8), where the data points cluster into different areas based on their quantum numbers. The S-P diagram gives the typical pulsation period and slopes of $\gamma$ Dor stars. For $l=1, m=1 \mathrm{~g}$ modes, the periods are between $0.3 \mathrm{~d}$ and $1.2 \mathrm{~d}$ with a slope around -0.03 , while for $l=2, m=2 \mathrm{~g}$ modes, the period is half that of the $l=1, m=1 \mathrm{~g}$ modes but the slope is similar. Both $l=2, m=2$ and $l=1, m=1$ $\mathrm{g}$ modes show a positive correlation between the slope and the mean period, which is an effect of rotation confirmed by the TAR simulations.

We obtain the near-core rotation rates, the asymptotic spacings, and the radial orders using the TAR. We find that the distribution of the near-core rotation rate shows a slowrotator excess, similar to the previous observations of the projected velocity $v \sin i$. There are more slow rotators than the angular momentum transfer models by Ouazzani et al. (2018) predicted, implying some additional mechanisms of angular momentum transfer are present inside these stars, or the effect of overshooting is significant. We obtained 11 fast rotators that show splittings, whose modes are $l=1, m=1$ and $l=1, m=0 \mathrm{~g}$ modes. Due to the rapid rotation, the splitting varies as a function of radial order. We find that the best-fitting TAR result can explain the period-spacing patterns but it can only partly explain the splittings. Surface modulations are found in 58 stars, with rotation rates close to the near-core rotation rates. Most $\gamma$ Dor stars rotate rigidly, with the near-core region rotating slightly faster, but not by more than $5 \%$.

Our observational sample is large enough to identify some outstanding problems in theoretical models of $\gamma$ Dor stars:

- Most stars in the $\gamma$ Dor instability strip do not show period spacing patterns, or their patterns are incomplete. Mode selection mechanisms for $\gamma$ Dor pulsations are needed.

- Concerning $\gamma$ Dor pulsation excitation, we confirm that a number of $\gamma$ Dor stars have hotter temperatures and also excite more radial orders than theoretically predicted.

- We observe that the near-core regions of $\gamma$ Dor stars rotate more slowely than expected, in disagreement with the theory. Two directions might be considered: fast rotation hinders g-mode pulsations, or our model for angular momentum transport is missing some key mechanisms.

\section{ACKNOWLEDGEMENTS}

The research was supported by an Australian Research Council DP grant DP150104667. Funding for the Stellar Astrophysics Centre is provided by the Danish National Research Foundation (Grant agreement no.: DNRF106). TVR has received funding from the European Research Council (ERC) under the European Union's Horizon 2020 research and innovation programme (grant agreement $\mathrm{N}^{\circ} 670519$ : MAMSIE) and from the KULeuven Research Council (grant C16/18/005: PARADISE). This work has made use of data from the European Space Agency (ESA) mission Gaia (https://www.cosmos.esa.int/gaia), processed by the Gaia Data Processing and Analysis Consortium (DPAC, https://www.cosmos.esa.int/web/gaia/ dpac/consortium). Funding for the DPAC has been provided by national institutions, in particular the institutions participating in the Gaia Multilateral Agreement.

\section{REFERENCES}

Abt H. A., Morrell N. I., 1995, ApJS, 99, 135

Asplund M., Grevesse N., Sauval A. J., Scott P., 2009, ARA\&A, 47,481

Balona L. A., 2013, MNRAS, 431, 2240

Balona L. A., Krisciunas K., Cousins A. W. J., 1994, MNRAS, 270, 905

Borucki W. J., et al., 2010, Science, 327, 977

Bouabid M.-P., Montalbán J., Miglio A., Dupret M.-A., Grigahcène A., Noels A., 2009, in Guzik J. A., Bradley P. A., eds, American Institute of Physics Conference Series Vol. 1170, American Institute of Physics Conference Series. pp 477-479 (arXiv: 0911.0775), doi:10.1063/1.3246547

Bouabid M.-P., Dupret M.-A., Salmon S., Montalbán J., Miglio A., Noels A., 2013, MNRAS, 429, 2500

Ceillier T., Eggenberger P., García R. A., Mathis S., 2013, A\&A, 555, A54

Chowdhury S., Joshi S., Engelbrecht C. A., De Cat P., Joshi Y. C., Paul K. T., 2018, Ap\&SS, 363, 260

Christophe S., Ballot J., Ouazzani R.-M., Antoci V., Salmon S. J. A. J., 2018, A\&A, 618, A47

Donati J.-F., Landstreet J. D., 2009, ARA\&A, 47, 333

Dupret M.-A., Grigahcène A., Garrido R., Gabriel M., Scuflaire R., 2005, A\&A, 435, 927

Eckart C., 1960, Hydrodynamics of oceans and atmospheres. Pergamon Press, Oxford, http://cds.cern.ch/ record/1975181

Eggenberger P., Montalbán J., Miglio A., 2012, A\&A, 544, L4

Fukuda I., 1982, PASP, 94, 271

Fulcher B. D., Jones N. S., 2017, Cell systems, 5, 527

Fulcher B. D., Little M. A., Jones N. S., 2013, Journal of the Royal Society Interface, 10, 20130048

Fuller J., Piro A. L., Jermyn A. S., 2019, MNRAS, 485, 3661

Gaia Collaboration et al., 2016, A\&A, 595, A1

Grassitelli L., Fossati L., Langer N., Miglio A., Istrate A. G., Sanyal D., 2015, A\&A, 584, L2

Groot P. J., Piters A. J. M., van Paradijs J., 1996, A\&AS, 118, 545

Guo Z., Gies D. R., Matson R. A., 2017, ApJ, 851, 39

Guzik J. A., Kaye A. B., Bradley P. A., Cox A. N., Neuforge C., 2000, ApJ, 542, L57

Hut P., 1981, A\&A, 99, 126

Kallinger T., et al., 2017, A\&A, 603, A13

Kaye A. B., Handler G., Krisciunas K., Poretti E., Zerbi F. M., 1999, PASP, 111, 840

Keen M. A., Bedding T. R., Murphy S. J., Schmid V. S., Aerts C., Tkachenko A., Ouazzani R.-M., Kurtz D. W., 2015, MNRAS, 454, 1792

Kirk B., et al., 2016, AJ, 151, 68

Koch D. G., et al., 2010, ApJ, 713, L79

Kurtz D. W., Saio H., Takata M., Shibahashi H., Murphy S. J., Sekii T., 2014, MNRAS, 444, 102

Ledoux P., 1951, ApJ, 114, 373

Lee U., Saio H., 1987, MNRAS, 224, 513 
Lee U., Saio H., 1997, ApJ, 491, 839

Li G., Bedding T. R., Murphy S. J., Van Reeth T., Antoci V., Ouazzani R.-M., 2019a, MNRAS, 482, 1757

Li G., Van Reeth T., Bedding T. R., Murphy S. J., Antoci V., 2019b, MNRAS, 487, 782

Maeder A., 2009, Physics, Formation and Evolution of Rotating Stars, doi:10.1007/978-3-540-76949-1.

Mathis S., Decressin T., Eggenberger P., Charbonnel C., 2013, A\&A, 558, A11

Mathur S., et al., 2017, ApJS, 229, 30

Mazumdar A., et al., 2014, ApJ, 782, 18

McQuillan A., Mazeh T., Aigrain S., 2014, ApJS, 211, 24

Mestel L., 1968, MNRAS, 138, 359

Mombarg J. S. G., Van Reeth T., Pedersen M. G., Molenberghs G., Bowman D. M., Johnston C., Tkachenko A., Aerts C., 2019, MNRAS,

Mosser B., et al., 2012, A\&A, 548, A10

Murphy S. J., Shibahashi H., Kurtz D. W., 2013, MNRAS, 430, 2986

Murphy S. J., Fossati L., Bedding T. R., Saio H., Kurtz D. W., Grassitelli L., Wang E. S., 2016, MNRAS, 459, 1201

Murphy S. J., Moe M., Kurtz D. W., Bedding T. R., Shibahashi H., Boffin H. M. J., 2018, MNRAS, 474, 4322

Murphy S. J., Hey D., Van Reeth T., Bedding T. R., 2019, MNRAS,

Ouazzani R.-M., Salmon S. J. A. J., Antoci V., Bedding T. R., Murphy S. J., Roxburgh I. W., 2017, MNRAS, 465, 2294

Ouazzani R.-M., Marques J. P., Goupil M., Christophe S., Antoci V., Salmon S. J. A. J., 2018, arXiv e-prints,

Papaloizou J., Pringle J. E., 1978, MNRAS, 182, 423

Pápics P. I., Tkachenko A., Aerts C., Van Reeth T., De Smedt K., Hillen M., Østensen R., Moravveji E., 2015, ApJ, 803, L25

Pápics P. I., et al., 2017, A\&A, 598, A74

Paxton B., Bildsten L., Dotter A., Herwig F., Lesaffre P., Timmes F., 2011, ApJS, 192, 3

Paxton B., et al., 2013, ApJS, 208, 4

Paxton B., et al., 2015, ApJS, 220, 15

Paxton B., et al., 2018, ApJS, 234, 34

Provost J., Berthomieu G., Rocca A., 1981, A\&A, 94, 126

Ramella M., Boehm C., Gerbaldi M., Faraggiana R., 1989, A\&A, 209, 233

Rieutord M., 2006, A\&A, 451, 1025

Royer F., Zorec J., Gómez A. E., 2007, A\&A, 463, 671

Saio H., 1982, ApJ, 256, 717

Saio H., Kurtz D. W., Takata M., Shibahashi H., Murphy S. J., Sekii T., Bedding T. R., 2015, MNRAS, 447, 3264

Saio H., Kurtz D. W., Murphy S. J., Antoci V. L., Lee U., 2018a, MNRAS, 474, 2774

Saio H., Bedding T. R., Kurtz D. W., Murphy S. J., Antoci V., Shibahashi H., Li G., Takata M., 2018b, MNRAS, 477, 2183

Shibahashi H., 1979, PASJ, 31, 87

Stumpe M. C., Smith J. C., Catanzarite J. H., Van Cleve J. E., Jenkins J. M., Twicken J. D., Girouard F. R., 2014, PASP, 126,100

Takada-Hidai M., Kurtz D. W., Shibahashi H., Murphy S. J., Takata M., Saio H., Sekii T., 2017, MNRAS, 470, 4908

Townsend R. H. D., 2003a, MNRAS, 340, 1020

Townsend R. H. D., 2003b, MNRAS, 343, 125

Townsend R. H. D., 2005, MNRAS, 360, 465

Triana S. A., Moravveji E., Pápics P. I., Aerts C., Kawaler S. D., Christensen-Dalsgaard J., 2015, ApJ, 810, 16

Van Reeth T., et al., 2015, ApJS, 218, 27

Van Reeth T., Tkachenko A., Aerts C., 2016, A\&A, 593, A120

Van Reeth T., et al., 2018, A\&A, 618, A24

Wade G. A., Petit V., Grunhut J. H., Neiner C., MiMeS Collaboration 2016, in Sigut T. A. A., Jones C. E., eds, Astronomical Society of the Pacific Conference Series Vol. 506, Bright Emissaries: Be Stars as Messengers of Star-Disk Physics. p. 207
Xiong D. R., Deng L., Zhang C., Wang K., 2016, MNRAS, 457, 3163

Zahn J.-P., 1992, A\&A, 265, 115

Zhang X. B., Fu J. N., Luo C. Q., Ren A. B., Yan Z. Z., 2018, ApJ, 865, 115 
We present four appendices: period-spacing patterns and TAR fittings, parameters of the $\gamma$ Dor stars, 11 rapidly rotating stars with rotational splittings, and 58 stars with surface rotation signals. The appendices are online only, here we only give the descriptions.

\section{APPENDIX A: PERIOD-SPACING PATTERNS AND TAR FITS}

We present the periodograms and period spacing patterns of $611 \gamma$ Dor stars. For each star, we show the periodogram with identified modes, the period spacing pattern(s) and the linear fitting(s), and sideways échelle diagram(s). We also show the TAR fitting and the posterior distributions of the near-core rotation rate and the asymptotic spacing.

\section{APPENDIX B: PARAMETERS OF THE $\gamma$ Dor STARS}

We list the observed and TAR fitting parameters of 611 $\gamma$ Dor stars in this paper. The parameters are: the Kepler magnitudes, the effective temperatures, the luminosities, the mode identifications $(k \equiv l-|m|, l$ is the angular degree and $m$ is the azimuthal order), mean pulsation periods $\langle P\rangle$, mean period spacings $\langle\Delta P\rangle$, slopes $\langle\Sigma\rangle$, asymptotic spacings $\Pi_{0}$, near-core rotation rates $f_{\text {rot }}$, the ranges of radial orders $n$, and ranges of spin parameters $s$. We also mark the stars which have short-cadence data or have $\mathrm{p}$ modes oscillations. The full version of this table can be found online.

\section{APPENDIX C: RAPIDLY-ROTATING STARS WITH ROTATIONAL SPLITTINGS}

We find 11 stars that rotate rapidly but still show rotational splittings. These stars are interesting because rotational splittings are rare among rapid rotators. The inclinations of these stars should be very small so the tesseral modes are seen, based on the amplitude distribution theory.

\section{APPENDIX D: SURFACE MODULATIONS OF 58 STARS}

58 stars show surface modulation signals. Most of them are caused by the surface activities while a few are classified as eclipsing binaries. Their orbital periods are short hence we assume their surfaces have been tidally locked.

This paper has been typeset from a $\mathrm{T}_{\mathrm{E}} \mathrm{X} / \mathrm{LAT}_{\mathrm{E}} \mathrm{X}$ file prepared by the author. 